\title{
Plants from disturbed savannah vegetation and their usage by Bakongo tribes in Uíge, Northern Angola
}

\author{
Anne Göhre ${ }^{1 *} \mathbb{D}$, Álvaro Bruno Toto-Nienguesse ${ }^{2}$, Macaia Futuro ${ }^{2}$, Christoph Neinhuis ${ }^{1}$ and Thea Lautenschläger $^{1}$
}

\begin{abstract}
Background: This study represents the first in-depth ethnobotanical study in the province of Uíge in northern Angola and documents the traditional knowledge of the Bakongo people living in the area. Due to deforestation and frequent fires, degraded savannahs dominate the landscape in the study region. Here we provide a list of useful plants from these savannahs including quantitative data about cultural importance of the respective species, aiming on the one hand to conserve the local knowledge and on the other hand to create a reliable basis for research projects in the region.
\end{abstract}

Methods: Field work was conducted in April and May 2014 in 5 municipalities of Uíge province. The study is based on 32 semi-structured and free-listing interviews, group discussions of varying scope and 14 field trips, involving a total of 82 informants. Throughout the course of the study herbarium specimens of the useful species were collected for later identification. Cultural importance index was applied to analyse the data sets recorded and to determine the best-known useful species in the region. All data sets were compared to the literature available for the region.

Results: The study documents a total of 498 citations for the use of 122 plants from 48 families, $34.0 \%$ of which were unknown according to the literature used for comparison. The high amount (71\%) of medical use-reports indicates that plants still play a crucial role in rural health care. We identified 14 plant species of special interest for pharmacological analysis. Species of highest cultural importance are Annona senegalensis Pers. and Sarcocephalus latifolius (Sm.) E.A., both of which are frequently found in disturbed savannahs.

Conclusions: The study points out the importance of savannahs even if degraded in terms of useful plants and provides a valuable addition to current knowledge of plant use in Northern Angola. This is not only essential for further studies, i.e. regarding pharmaceutical agents, but also for the design of a planned botanical garden of the University Kimpa Vita in Uíge, which aims at communicating the findings to the local people.

Keywords: Ethnobotany, Disturbance vegetation, Angola, Bakongo people, Traditional knowledge

\section{Background}

Due to its botanical and cultural diversity, covering vegetation zones from tropical cloud forests in the north to the Namib Desert in the south [1] and many ethnic groups [2], Angola is a promising target for ethnobotanical research.

\footnotetext{
* Correspondence: anne.goehre@tu-dresden.de

${ }^{1}$ Institut für Botanik, Technische Universität Dresden, D-01062 Dresden,

Germany

Full list of author information is available at the end of the article
}

Previous studies already aimed at documenting ethnobotanical knowledge in Angola and preserving it for future generations. Leyens and Lobin [3] present portraits of Miombo and Mopane plant species and their uses, while Costa and Pedro [4] focus on medical plants and summarize data collected from across the country. Latham [5, 6], Kembelo [7] and Makumbelo [8] offer comparable information for the Bas-Congo-Province in the Democratic Republic of Congo, adjacent to the study area. However, systematic and comprehensive ethnobotanical analyses regarding the cultural and botanical diversity of the country 
are still scarce. The above-mentioned publications primarily document and propagate traditional knowledge, e.g. to improve health care for the local population, but do not include quantitative data and in some cases don't give specific information about the ethnic groups using the plants. A recent study of Urso et al. [9] offers first quantitative data for the province of Namibe in the south-west of the country. Their survey emphasizes the importance of plants to both cultural identity and livelihood strategies in the area and identifies a high amount of species that were until then not known for their ethnobotanical importance. No comparable data are available for the north of the country. Due to 40 years of Civil War and War of Independence, a lot of research needs to be done in this field.

It was the aim of this study to document traditional knowledge about useful plants in the Province of Uíge in Northern Angola. The examined municipalities are widely dominated by savannah vegetation, severely degraded by frequent fires $[1,10]$. Several ethnobotanical studies worldwide have stated that those disturbed areas are far more important to the local people than would be expected by means of species richness [11, 12]. Disturbed habitats are often found in the vicinity of human settlements, facilitating accessibility $[12,13]$, and contain a high percentage of weeds that are assumed to be of high medical value $[14,15]$. The goal of this first indepth ethnobotanical assessment of the region was to provide a list of the useful plants from savannahs also including quantitative data about cultural importance of species. By this means, the study aims to conserve local knowledge for future generations, to make it accessible for projects such as a planned botanical garden and to interpret it in the context of anthropogenic disturbance. Apart from that, our documentation intends to provide a reliable basis for future ethnobotanical and ethnopharmacological research in the region and to identify promising targets for such projects.

\section{Study area}

Field work was carried out in the Province of Uíge in the north of Angola (Fig. 1). The study area extends in the range of $\mathrm{S}^{\circ} 55^{\prime} 24.91^{\prime \prime}$ to $\mathrm{S}^{\circ} 7^{\circ} 57^{\prime} 03.7^{\prime \prime}$ and $\mathrm{E} 14^{\circ}$ $36^{\prime} 14.7^{\prime}$ to $\mathrm{E} 15^{\circ} 30^{\prime} 27.02$ ", mainly in the municipality of Uíge. Other municipalities covered by the study are Negaje, Quitexe, Mucaba and Ambuíla.

\section{Climate and vegetation}

Uíge Province is characterised by tropical wet and dry or savannah climate, classified by Köppen-Geiger as Aw $[16,17]$. Despite four months of dry season extending from June to September [18], the surroundings of Uíge City were originally covered by closed forest formations and forest savannah mosaic [19]. The development of forests is favoured by a local climate in the neighbouring mountain chains of Serra do Uíge, Serra do Pingano and Serra Quibinda, where humid air masses condense during dry season $[10,20]$.

The forests in Uíge are under huge anthropic pressure: Timber exploitation, agriculture (mainly subsistence farming and slash-and-burn agriculture), urbanization and fragmentation of the habitat due to road construction lead to the degradation and loss of this habitat. The area of degraded savannahs increases, resulting in a mosaic of remaining cloud forests, secondary coffee forests and savannahs $[1,10,19]$.

As illustrated in Fig. 1, most of the data sampling points are located in areas of potentially closed formations of semi-deciduous rainforest or evergreen cloud forests or in areas of secondary coffee forests and Zambesi savannahs. The actual vegetation is characterized by degraded savannahs, as seen in Fig. 2, which are widely dominated by the grass genus Hyparrhenia. Pyrophytic, rhizomatic herbs such as Pteridium centrali-africanum and Aframomum alboviolaceum and small, fire resistant shrubs such as Psorospermum febrifugum, Bridelia ferruginea and Hymenocardia acida are commonly found. The vegetation as well as interviews and personal observations lead to the conclusion that these savannah habitats are burnt on a regular basis. Different reasons for those large scale savannah fires were mentioned during interviews: Fires are meant to keep roads clear, to improve accessibility of grassland (e.g. to collect fruits of Aframomum alboviolaceum), to reduce agricultural pests, to create pasture land, to locate enemies earlier (during civil war) or are started without reasons or as a game.

Especially in interviews it was not always possible to differentiate between savannahs and other disturbed habitats in the vicinity of human settlements, such as village outskirts, fields, forest edges and recently burnt savannahs or forest patches. Also, field excursions sometimes included transition zones to those habitats, where vegetation is influenced by forest species, introduced or cultivated plants and/or ruderal species.

\section{Population}

The province of Uíge covers an area of $58.698 \mathrm{~km}^{2}$ and counts about 1.4 million inhabitants [21]. The majority of the local population belongs to the Bakongo ethnic group. This Bantu group lives in an area that covers parts of what is now Angola, Democratic Republic of the Congo, Republic of the Congo and Gabon. They are united by the Bantu-language Kikongo, although the wide distribution, different colonial powers, transregional trade and migration have lead to a great diversity in spoken and written forms of this language [22]. In Angola most persons additionally speak the official language Portuguese.

In the rural areas involved in the study, agriculture is the main source of food and income. Small subsistence 


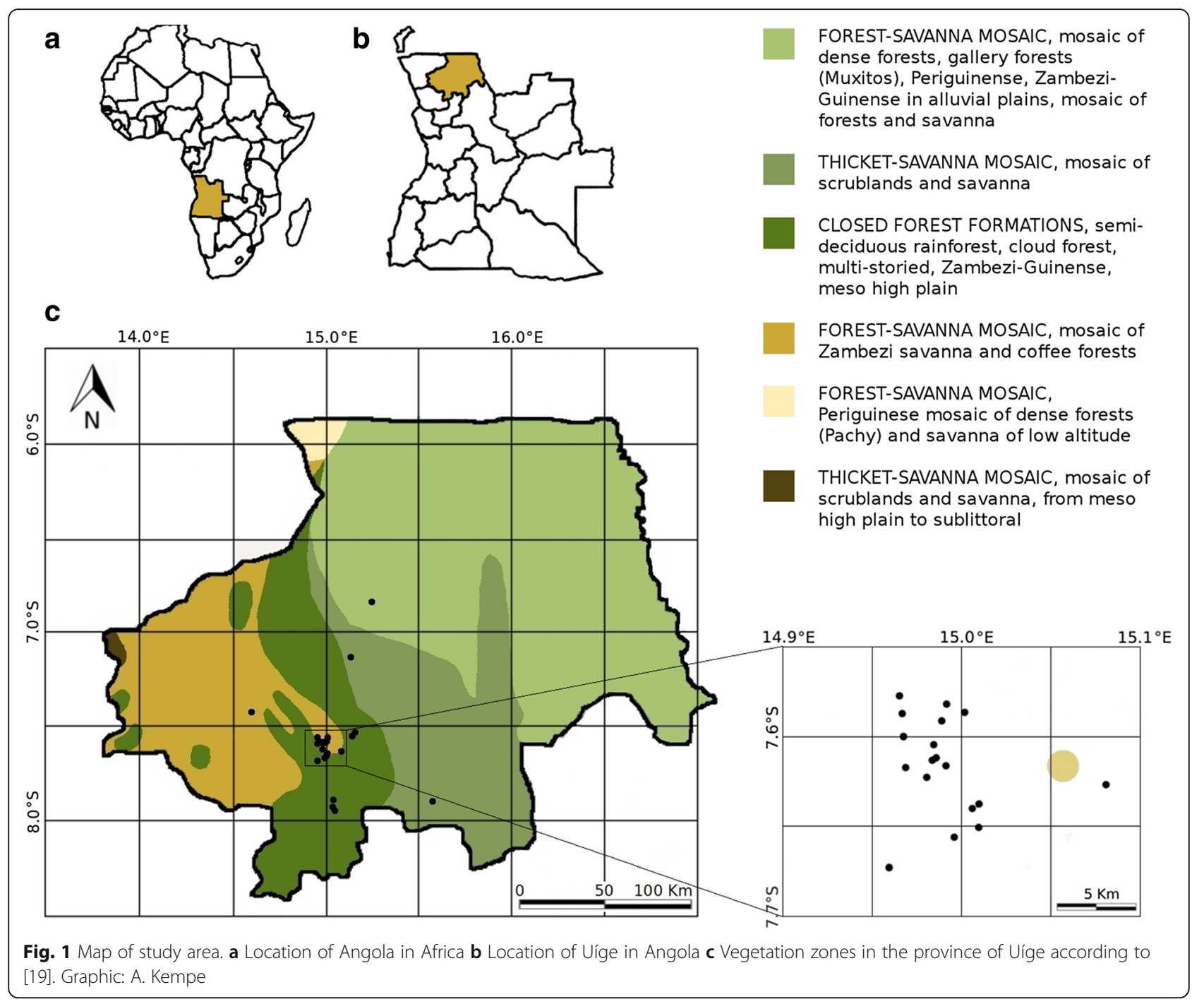

family farms are prevailing [1], information on field size ranges between 1.4 ha and 2.25 ha [23, 24]. Most smallholders practise shifting cultivation. As mechanization is uncommon, the land is mostly prepared manually and fire is often used to remove biomass (slash-and-burn agriculture) [1].

Western medical health care is scarce, especially in rural areas [25]. The under-five mortality rate in Angola is the highest in the world, with 157 deaths per 1000 live births [26]. Curent economic problems due to the low oil price might aggravate the situation. It is assumed that importance of traditional healers and herbal medicines especially for rural people is high and might even increase in the coming years $[9,27]$.

\section{Methods}

Field work was conducted between April 6th and May 24th, 2014. The study is part of an academic cooperation between Technische Universität Dresden and Universidade

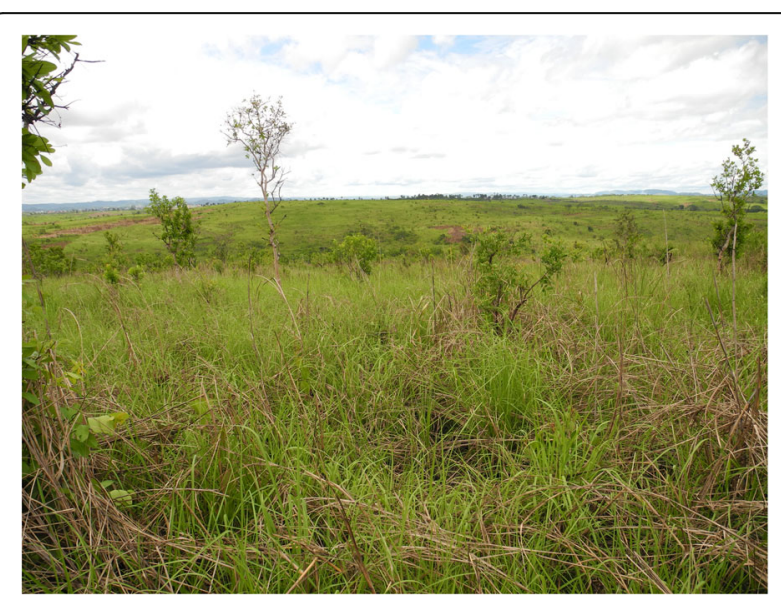

Fig. 2 Degraded savannah landscape close to the city of Uíge. Study site close to the experimental farm of Kimpa Vita University 
Kimpa Vita. Permits for research, collection and export of voucher specimens were obtained from Instituto Nacional da Biodiversidade e Áreas de Conservação of the Ministério do Ambiente da República de Angola and from Direcção Provincial da Agricultura, Desenvolvimento Rural e Pescas of the Governo Provincial do Uíge.

Prior to interviews and field excursions all participants in the study were informed about the project and their rights and local authorities were asked for permission to work within the community and the surroundings (prior informed consent). Communication was mainly conducted in Portuguese. If necessary, Angolan co-workers or village residents translated into Kikongo. The methods used for ethnobotanical data collection included semi-structured interviewing, free-listing, group discussions regarding plant identification and field excursions. Data sets collected included the vernacular name of the plant in Kikongo and/or Portuguese, usage and the plant parts used as well as preparation and administration techniques. Data sets collected during interviews were only included in the analysis if they could be assigned to plants identified during field trips.

During field excursions and subsequent to interviews, herbarium specimens were collected for later identification. All voucher specimens are deposited in the Herbarium Dresdense (DD) of the Institute of Botany, Technische Universität Dresden, Germany. As soon as suitable conditions are established, type species and duplicates of the collection will be deposited at Universidade Kimpa Vita, Uíge, Angola. Identification of plant species was carried out at the Institute for Botany of TU Dresden. If it was not possible to collect herbarium specimens, photographs of the plants were used. The following floristic works were consulted for identification: Conspectus Florae Angolensis [28-30], Plantas de Angola [31], Flora of Tropical West Africa [32-36], Flore Analytique du Bénin [37] and Flora Zambesiaca [38]. Additional information was retrieved from Kew Herbarium Catalogue [39], Herbario LISC [40] and Herbarium Dresdense.

Because of time constraints, not all plants were found with flowers and fruits, impeding the determination to species level. The associated use-reports were only included in the results if the specimen was at least determined to family level.

In total, 41 informant groups were involved in the study. These groups were made up of 82 individuals between the ages of 23 and 80 years, with $56 \%$ of informants being women. We conducted 32 interviews and 14 field trips with these informant groups. Group size varied between one and nine persons in interviews, with a mean value of 2.3. In field excursions, group size varied between one and four participants, with an average value of 1.8. Whether field excursions and interviews were carried out with single persons or groups was mainly influenced by decisions of local authorities.

To establish contact with potential informants, local authorities were asked to suggest persons with different backgrounds that might participate in the study. The great majority $(74 \%)$ of informants were farmers who were known within the community for having knowledge about the use of plants. $10 \%$ of participants belonged to the group of local authorities, who were sometimes also involved in farming activities. A further $7 \%$ of participants were traditional healers or midwives, $6 \%$ workers or employees (non-farming activity), while teachers within the villages represented $3 \%$. To quantify ethnobotanical data, the information regarding useful plants was organised in use-reports. Use-reports follow the basic structure of 'informant [group] i mentions the use of species $\mathrm{s}$ in the use-category $\mathrm{u}^{\prime}[41,42]$. Usecategories were adopted from the data received during the interviews and included: nutrition/food, medicine, tea, ornamental, ritual/magic, forage, fibres, handicraft/ building material and fuel. Use categories mentioned less than 4 times (e.g. fish poison, candles) were summarised in the residual category "others".

Use reports were analysed calculating the number of use-reports (NUR) and the cultural importance index (CI) (Formula 1). The NUR is one of the most commonly used tools to measure the cultural importance of plants [41]. It is calculated by firstly summing the number of informants who mention each use-category for the species and secondly summing the values of each use-category [41]. The CI was introduced by Tardío and Pardo-de-Santayana [41]. It is comparable to the use value, which is widely applied in ethnobotanical research $[13,43]$. Unlike NUR, it is not influenced by the total number of informants and therefore allows the comparison of data between different studies. As informants were mostly interviewed in groups and use reports were only recorded once per group, we used the number of the interviewed groups substitutional for the total number of informants in our calculation.

To give more accurate information about the use of plants, large use-categories were divided into subcategories or usages. In the medical use-category, subcategories were determined by the illness or symptom treated with the plant. Subcategories were documented without further grouping of the cited usages or illnesses. For food plants, subcategories were defined by the plant part and the preparation method used. Subcategories mentioned during the interviews included: Raw fruits (eaten fresh or dry), cooked fruits, cooked leaves (eaten as vegetable), cooked tubers and raw stem.

If one informant mentioned the use of a species in two subcategories belonging to the same use-category, those use-reports were counted as one data-set in the use- 
category for calculation of NUR and CI. E.g., if one informant mentioned the use of a species against abdominal pain and diarrhoea, those use-reports counted as one data-set in the category "medicine". This was necessary because many use-reports, especially for medical uses, were closely related, which could result in an overestimation of cultural importance. Because of the introduction of subcategories, NUR per species does not always equal the sum of all use-reports listed in the subcategories.

$$
\begin{aligned}
& N U R_{s}=\sum_{u=u_{1}}^{u_{N C}} \sum_{i=i_{1}}^{i_{N I}} U R_{u i} \\
& C I_{s}=\sum_{u=u_{1}}^{u_{N C}} \sum_{i=i_{1}}^{i_{N I}} \frac{U R_{u i}}{N I}
\end{aligned}
$$

Formula 1: (A) Calculation of number of use-reports and (B) calculation of cultural importance index according to [41]. $N U R=$ Number of use-reports, $C I=$ cultural importance index, $s=$ species, $u=$ use-category, $i=$ informant, $N I=$ total number of informants, $N C=$ number of use categories, $U R=$ use-report.

To evaluate the increase of knowledge through the study, all data sets were compared to available literature. For use-reports referring to medical treatments Neuwinger [44], for all other applications Latham [45] and Latham and Konda ku Mbuta [5] were used for comparison. To estimate the potential for further medical studies, a PubMed inquiry (http://www.ncbi.nlm.nih.gov/pubmed) was carried out in June 2015 for each species to determine if medical studies have already been carried out.

\section{Results}

The study documents a total of 498 different use-reports for 122 plants from 48 families. All use-reports are summarised and provided in Table 1 . The most commonly used plant families are Fabaceae (13.1\%), Asteraceae (13.1\%), Euphorbiaceae (6.6 \%), Lamiaceae (6.6 \%) and Malvaceae (5.7\%), all of which are widely distributed in Angola [31].

On average, 4.1 different use-reports per species were documented, $72.1 \%$ of which refer to medical treatments. $10.6 \%$ of uses refer to food plants. Other use categories covered are handicraft and building material (3.6\%), tea (non medical, $2.6 \%$ ), fuels (1.8\%), forage plants (1.8 \%), ritual uses $(1.8 \%)$, source of fibers $(1.4 \%)$, ornamentals (1.0\%), dyes (1.0\%), and others (2.2\%).

Of the documented plants, $41.3 \%$ were shrubs or trees, $9.1 \%$ subshrubs, $24.8 \%$ perennial herbaceous plants, $13.2 \%$ annuals and $11.6 \%$ annual or perennial climbers. Due to regular fires many species that are documented in the literature as trees, showed a shrubby habit in the study area. The most commonly used plant parts are leaves or fronds (224 citations for 78 species), fruits (73 citations for 35 species, including seeds) and underground organs, such as roots, root tubers and rhizomes (75 citations for 28 species).

NUR and CI were calculated for each species to evaluate the importance of a particular plant species and to standardize the data. NUR varies between 1 and 16, with an average value of 3.63. The related value of CI covers a range from 0.02 to 0.39 with an average of 0.09 . Table 2 summarises the ten highest-ranking species. The highest values were reached by Annona senegalensis Pers. and Sarcocephalus latifolius (Sm.) E.A. Bruce with NUR $=16$ and $\mathrm{CI}=0.39$.

For all specimens determined to species level, literature comparison was carried out to emphasize hitherto unknown use-reports. $7.6 \%$ of citations referred to species not documented in the ethnobotanical literature used for comparison and $26.6 \%$ of the reports added new uses to species that were already known for their ethnobotanical relevance.

\section{Medicinal plants}

The high percentage of medical use-reports allowed further analyses of the diseases treated with traditional medicine. As can be seen in Fig. 3, medical plants are used against both specific diseases, such as typhoid fever or diabetes, and widespread symptoms like abdominal pain. For reasons of clarity, some symptoms listed separately in Table 1, are merged in Fig. 3 by means of the body systems affected. E. g., "digestive tract diseases" includes constipation, diarrhoea and nausea; "gynaecologic disorder" merges menstrual disorders with fertility and pregnancy disorders. As abdominal pain is a widespread symptom which might be caused by several diseases, this use category was kept separately from related categories, such as digestive tract or gynaecological disorder, hepatitis or urinary tract infections, if no clear attribution was made by informants. The most frequently listed areas of application are abdominal pain (55 use-reports), digestive tract diseases (28 UR), childhood diseases (28 UR), rheumatic or muscle pain (25 UR) and fevers (25 UR). Two diseases mentioned in interviews could not be identified and were therefore not translated. Baço refers to a disease affecting mostly children, including symptoms that might be related to splenomegaly after malaria infection. Gota would be literally translated as gout, but is commonly described as a childhood disease with epileptic symptoms.

The most frequently cited preparation methods for medical plants are decoctions (72 citations) and freshly crushed material, as it is used for dermal administration or to extract the sap from a tissue (71 citations). Furthermore, use-reports include infusions (59 citations), 
Table 1 Overview of the documented useful plant species and their uses sorted by plant families

\begin{tabular}{|c|c|c|c|c|c|c|c|c|}
\hline$\overline{U C^{a}}$ & Subcategory, explanation of usage & $P P^{b}$ & Preparation, Explanations & Administration & $\mathrm{N}^{c}$ & $\mathrm{Cl}^{\mathrm{d}}$ & $L^{e}$ & $\mathrm{DB}^{f}$ \\
\hline \multicolumn{9}{|c|}{ ACANTHACEAE } \\
\hline \multicolumn{5}{|c|}{ Acanthus montanus (Nees) T.Anderson; +; 042677} & 1 & 0.02 & & $M, C$ \\
\hline M & Skin disease & L & Infusion & Dermal & 1 & & o & \\
\hline \multicolumn{5}{|c|}{ Brillantaisia owariensis P.Beauv.; Lemba-lemba; + 044075} & 4 & 0.10 & & M \\
\hline M & Cardiac disease & L & Decoction (with sugar) & Oral & 1 & & o & \\
\hline M & Childhood disease & L & & Bath & 2 & & - & \\
\hline M & Blood pressure (high or low) & L & Directly consumed & Oral & 1 & & o & \\
\hline M & Abdominal pain & L & Directly consumed & Oral & 2 & & + & \\
\hline M & Childhood disease (Gota) & L & Cold water extract & Oral & 1 & & - & \\
\hline M & Eye disease & L & Directly consumed & Oral & 1 & & - & \\
\hline \multicolumn{9}{|c|}{ AMARANTHACEAE } \\
\hline \multicolumn{5}{|c|}{ Dysphania ambrosioides (L.) Mosyakin \& Clemants; Santa Maria, Nkavua; * (Chenopodium ambrosioides), 042698} & 10 & 0.24 & & $\mathrm{M}, \mathrm{C}$ \\
\hline M & Abdominal pain & L & & Enema & 1 & & o & \\
\hline M & General & L & Directly consumed & Oral & 2 & & - & \\
\hline M & Respiratory disease & L & Infusion & Oral & 1 & & + & \\
\hline M & Backache and rheumatic pain & L & $\begin{array}{l}\text { With Milletia versicolor, Ocimum gratissimum } \\
\text { and Persea americana }\end{array}$ & Steam Bath & 1 & & + & \\
\hline M & Fever & L & $\begin{array}{l}\text { With Cyperus sp., Xylopia aethiopica, Monodora } \\
\text { myristica, Kafuke (Asteraceae indet.), }\end{array}$ & Enema & 1 & & + & \\
\hline M & Gynaecological disorder & L & With Chromolaena odorata and O. gratissimum & & 1 & & + & \\
\hline M & Malaria & L & $\begin{array}{l}\text { With X. aethiopica, Kafuke (Asteraceae indet.), } \\
\text { M. myristica, O. gratissimum }\end{array}$ & & 1 & & o & \\
\hline M & Childhood disease (Growth disorders) & L & With Ageratum conyzoides and O. gratissimum & Dermal & 1 & & o & \\
\hline M & Diarrhoea & L & $\begin{array}{l}\text { (A) Directly consumed (B) with X. aethiopica, } \\
\text { Kafuke (Asteraceae indet.), M. myristica, } \\
\text { O. gratissimum }\end{array}$ & Oral & 2 & & o & \\
\hline \multicolumn{9}{|c|}{ ANACARDIACEAE } \\
\hline \multicolumn{5}{|c|}{ Lannea cf. antiscorbutica (Hiern) Engl.; Nkumbi; +; F10-1961, 043178} & 4 & 0.10 & & - \\
\hline M & Toothache & B & $\begin{array}{l}\text { (A) Decoction of crushed bark (B) with } \\
\text { Carica papaya }\end{array}$ & (A) Applied to tooth & 2 & & / & \\
\hline M & Pulled muscles, fractures & B & Decoction of crushed bark & $\begin{array}{l}\text { Applied externally and left } \\
\text { to dry, forms cast }\end{array}$ & 3 & & / & \\
\hline
\end{tabular}


Table 1 Overview of the documented useful plant species and their uses sorted by plant families (Continued)

ANISOPHYLLACEAE

Anisophyllea cf. quangensis Engl. ex Henriq; Mfungua, Bilasoba; +; 043115
M Skin disease
Balm is made after bark is removed
$N \quad$ Fresh fruit

$$
\text { Directly consumed }
$$$$
\text { F } \quad \text { Directly consumed }
$$

Dermal

0.05

ANNONACEAE

Annona senegalensis Pers.; Nlolo, Nlolo kambulu, Nlolopolo, Nloloa pequena, Mfuilu; +; 043115

$\begin{array}{llll}\text { M } & \text { Menstrual disorder } & R & \text { Decoction } \\ M & \text { Haemorrhoids } & R & \text { Crushed root } \\ M & \text { Fertility } & R, L, B & \text { Infusion } \\ M & \text { Hernia } & R & \text { Decoction } \\ M & \text { Leg pain } & R, L & \text { Infusion } \\ M & \text { Abdominal pain } & R, L & \text { Decoction } \\ M & \text { Worms } & R, L & \text { Infusion } \\ M & \text { Respiratory disease } & & \text { With Aframomum alboviolaceum } \\ N & \text { Fresh fruit } & \text { F } & \\ T & & L & \end{array}$

Monodora myristica (Gaertn.) Dunal; Peve, Gipeve, Gipehe; +; 042679

\begin{tabular}{|c|c|c|c|}
\hline M & Cough & S & Directly consumed \\
\hline M & Malaria & S & $\begin{array}{l}\text { With } X \text {. aethiopica, Kafuke (Asteraceae indet.), } \\
\text { D. ambrosioides, O. gratissimum }\end{array}$ \\
\hline M & Diarrhoea & S & $\begin{array}{l}\text { with } X \text {. aethiopica, Kafuke (Asteraceae indet.), } \\
\text { D. ambrosioides, O. gratissimum }\end{array}$ \\
\hline M & Abdominal pain & L, S & Sometimes on combination with other species \\
\hline M & Backache & S & $\begin{array}{l}\text { With Ochna cf. afzelii subsp. mechowiana., } \\
\text { M. myristica and Kupidi (Piper sp.) }\end{array}$ \\
\hline M & Fever & S & $\begin{array}{l}\text { With Cyperus sp., X. aethiopica, D. ambrosioides, } \\
\text { Kafuke (Asteraceae indet.), }\end{array}$ \\
\hline M & Worms & S & $\begin{array}{l}\text { Infusion with other species, e.g. Kupidi (Piper sp.), } \\
X \text {. aethiopica and Ndungu za nzó (Aframomum sp }\end{array}$ \\
\hline
\end{tabular}

APIACEAE

Steganotaenia araliacea Hochst.; Mumvumbimvumbi; +; 043212
M Thrombosis
L Decoction
M Fertility
B, R Decoction of 1,5 spoon of powder in 1 I of water

Oral

Rectal

Oral

Oral, 2 cups daily

Oral

Oral or enema

Oral

Oral

$0.20 \quad M, C$

Enema

Enema

Oral

0.02

Oral, 2 cups daily
$+$

o 
Table 1 Overview of the documented useful plant species and their uses sorted by plant families (Continued)

APOCYNACEAE

Landolphia owariensis P.Beauv.; Macongue, Makonge; +; 043969
$\mathrm{N} \quad$ Fresh fruit
F Directly consumed

AsteRACEAE

Acanthospermum sp.; Makoloko; F09-1725
M Headache
P Directly consumed

Ageratum conyzoides (L.) L.; Kambwa katela, Mbokatela, Mbukata;+; 043150
M Fever
M Eye disease
P Decoction
M Childhood disease (Growth disorders)
Leaf sap from crushed leaves
M Abdominal pain
L $\quad$ With O. gratissimum and D. ambrosioides

Baccharoides guineensis (Benth.) H.Rob;; Matita, Matitita, + (as Vernonia guineensis Benth.); 043110
M Worms
Cold water extract from crushed root tubers
M Childhood disease (Baço)
M Wounds
M Hernia
Directly consumed (peeled root tubers)
Directly consumed (peeled root tubers)
M Abdominal pain
Directly consumed (peeled root tubers)
Directly consumed (peeled root tubers)

Bidens pilosa L.; Potajambua; +; 042705

L

Chromolaena odorata (L.) R.M.King \& H.Rob.; Mobutu, Kongo dia sika, Mululusaire; - (*); 042708
M Gynaecological disorder
L $\quad$ With O. gratissimum and D. ambrosioides
M Abdominal pain
$L \quad$ (A) Cold water extract of crushed leaves
(B) crude crushed leaves
M Wounds
$\begin{array}{ll}\text { L } & \text { Crushed leaves } \\ \text { P } & \text { Planted in Manihot esculenta field }\end{array}$
Ot Soil fertility

Crassocephalum rubens (Juss. ex Jacq.) S. Moore; Bungudia; +; 044078
$\mathrm{N}$ Cooked leaves
Cooked and eaten as a vegetable
For rabbits and pigs

Emilia coccinea (Sims) G.Don; Malalulalu; +; 044086, 044087

$\begin{array}{clll}M & \text { Wounds } & L & \text { Leave sap from crushed leaves } \\ \text { Fo } & & L & \text { For rabbits } \\ M & \text { Eye disease } & L & \text { Leave sap from crushed leaves }\end{array}$

(A) Oral/enema (B)

Compress (abdomen)

Dermal, applied to wound

6

Chew

Bath

Applied to eye

Dermal

Enema

Oral

Oral

Oral

Oral

Dermal, applied to wound

Dermal, applied to wound

Rubbed into eyes 
Table 1 Overview of the documented useful plant species and their uses sorted by plant families (Continued)

Galinsoga quadriradiata Ruiz \& Pav.; Kabuata branca; (*); 043141

M Hepatitis L Decoction

Gymnanthemum cf. glaberrimum (Welw. ex O.Hoff.) H.Rob.; Nsalu; Kisalu; 044039

$\begin{array}{llll}\text { M Anaemia } & R & \text { Decoction of crushed root } \\ \text { M } & \text { Scabies } & L & \text { Ash } \\ \text { M } & \text { Backache } & R & \text { Cold water extract } \\ M & \text { Worms } & R & \text { Cold water extract } \\ M & \text { Abdominal pain } & \text { R, L } & \text { (A) Crushed root (B) Infusion }\end{array}$

Helichrysum mechowianum Klatt; + (var. mechowianum); 044088
Ot Toilet paper
$\mathrm{L}$
-; Kafuke; 044034
M Abdominal pain
L Sometimes with e.g. X. aethiopica,
M. myristica
M Childhood disease (light stomach pain)
L
With D. ambrosioides, X. aethiopica
M. myristica, O. gratissimum
M Diarrhoea
R With Cyperus sp., X. aethiopica,
D. ambrosioides, M.myristica
M Fever
M Malaria
M Fertility
M Worms
L $\quad$ With Urena lobata

Melanthera scandens (Schumach. \& Thonn.) Roberty; Kalahi, Kalau, Makaila; +; 042782, 042778
M Wounds

$\mathrm{L}$

Pleiotaxis rugosa O.Hoffm.; Ntelamakatexe, Kakatiana; 044020
M Abdominal pain
$L, R \quad$ Directly consumed or infusion
M Diarrhoea
$L, R \quad$ Directly consumed (root) or infusion
(leaves), masticate root

Polydora serratuloides (DC.) H.Rob.;+ (listed as Vernonia perrottetii Sch. Bip. ex Walp.); 042793
$\mathrm{O}$
P Planted on graveyards

Tithonia diversifolia (Hemsl.) A.Gray; Mululula; *; 044090

M Abdominal pain

L Crushed leaves

Vernonella subaphylla (Baker) H.Rob. \& Skvarla; Makutula; + (listed as Vernonia subaphylla Baker); 044024, 042795, 042797
M Skin disease (furuncle)
Infusion

Oral

Oral

Derma

Oral

Oral

(A) Dermal (B) Oral

0.02

M

0.10

$-$

$+$

$-$

$+$

0.02

0.17

Enema

Enema

Enema

Enema

Enema

Dermal, applied to wound

Oral, not during pregnancy

Compress (abdomen)

Oral

$\begin{array}{rrr}0.05 & & M, C \\ & + & \\ 0.07 & & - \\ & / & \\ & / & \\ & & \\ 0.02 & & - \\ & / & \\ 0.02 & & M, C \\ & + & \\ 0.12 & & - \\ & / & \end{array}$


Table 1 Overview of the documented useful plant species and their uses sorted by plant families (Continued)

BIXACEAE

Bixa cf. orellana L.; Ndalamuenga; *; F03-131

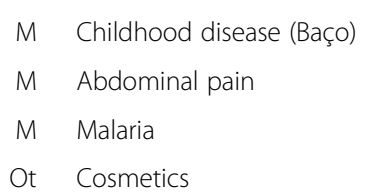

Decoction

Directly consumed or cold water extract

Decoction

BURSERACEAE

Used for red colour

Canarium schweinfurthii Engl.; Mbidi, Gimbidi; +; F09-1730, F09-1734

$\begin{array}{llll}\text { N Cooked fruit } & \text { F } & \text { Left in warm water for } 1 \mathrm{~h} \text { or until pulp softens } \\ \text { M Respiratory disease } & \text { Re } & \\ \text { M Toothache } & \text { L } & \text { Decoction with Psidium guajava and Alchornea cordifolia } \\ \text { M Abdominal pain } & \text { Re, R } & \text { (A) heat resin and mix with palm oil, } X \text {. aethiopica and } \\ \text { M Nightmares } & \text { Re } & \text { M. myristica (B) clean (and crush) crude root } \\ \text { Ot Candles } & \text { Re } & \text { Used as wax substitute }\end{array}$

Dacryodes edulis (G.Don) H.J.Lam; Safueiro, +, F02-1181

$N$ Cooked fruit $\quad F \quad$ Heated in water or in pan until pulp softens

\section{CANNACEAE}

Canna indica L.; Chala (verde), Cholo; *; 042701

$\begin{array}{lll}\text { M Thorax pain } & \text { L } & \text { Whole leaves } \\ \text { H } & \text { S } & \text { For rattles } \\ \text { CARICACEAE } & & \\ \text { Carica papaya L; Mamoeiro; *; F10-1978 } & & \\ \text { M Abdominal pain } & L & \text { Crushed leaves } \\ \text { M Nausea } & \text { L } & \text { Crushed leaves } \\ \text { M Toothache } & \text { R } & \text { Decoction, (sometimes with Lannea antiscorbutica) }\end{array}$

CARYOPHYLLACEAE

Drymaria cordata (L.) Willd. ex Schult.; Lumpwua; +; 042678
M Hepatitis
L, P Crushed material

Oral

Oral

Oral
Rubbed on breast, inhalatio
Rinsed in mouth
(A) Add to food (B) Oral
or enema

Inhalation

\subsection{7}

$M, C$

o

$-$

$+$

0.15

M, C

$+$

$-$

$+$

-

o

0.05

M, C

0.05

M, C

Compress at abdomen

Compress at abdomen

Compress at abdomen

Rinsed in mouth

$+$

0

$+$

0.02

Dermal or enema

\section{COMMELINACEAE}

Commelina diffusa Burm.f.; Ndakalaka; +; 044029 
Table 1 Overview of the documented useful plant species and their uses sorted by plant families (Continued)

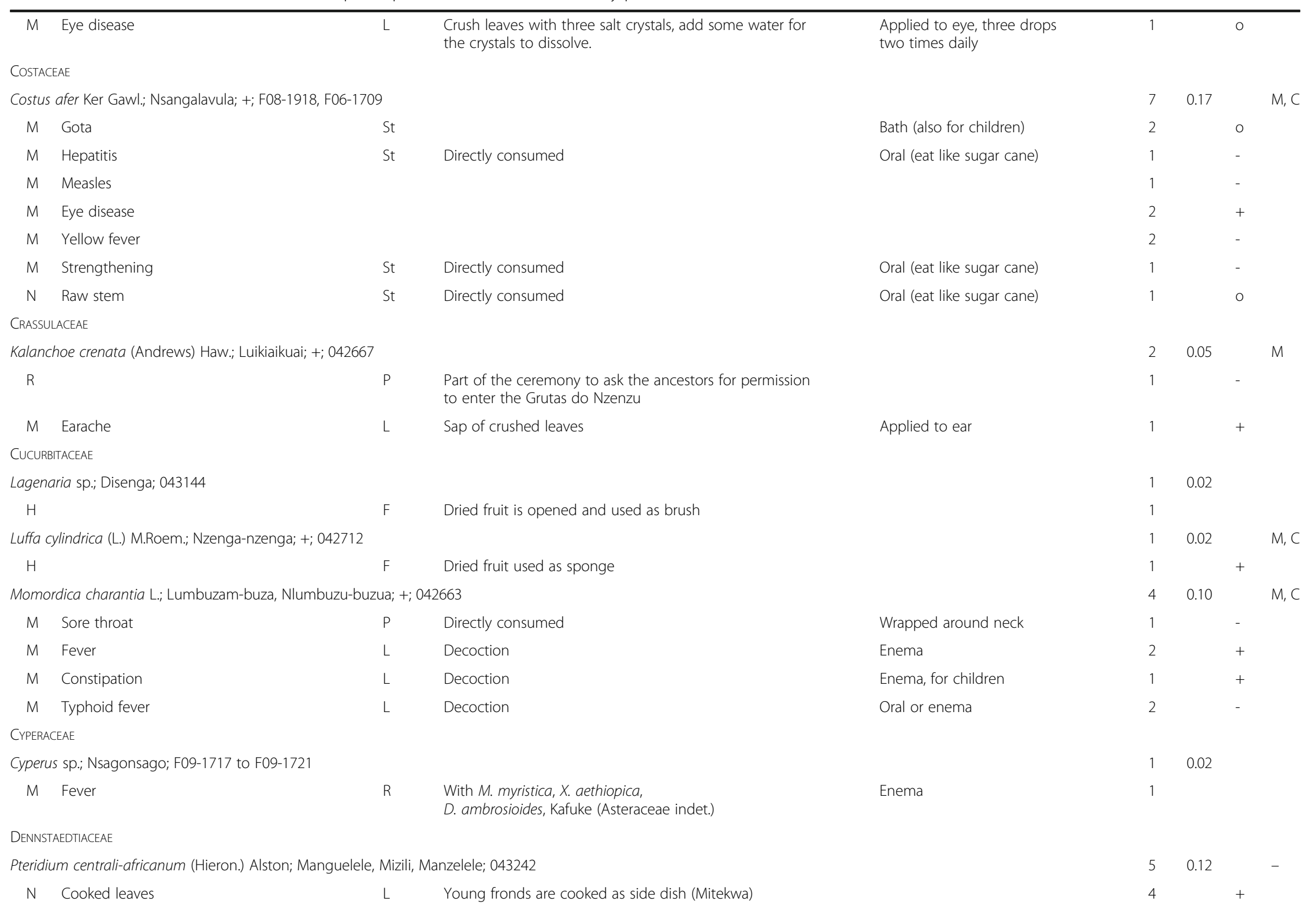


Table 1 Overview of the documented useful plant species and their uses sorted by plant families (Continued)

\begin{tabular}{|c|c|c|c|c|c|c|c|c|}
\hline M & Epilepsy & $\mathrm{L}, \mathrm{Rh}$ & Decoction & Bath or enema & 1 & & - & \\
\hline \multicolumn{9}{|c|}{ DIOSCOREACEAE } \\
\hline \multicolumn{4}{|c|}{ Dioscorea dumetorum (Kunth) Pax; +; 044023} & & 1 & 0.02 & & $\mathrm{M}, \mathrm{C}$ \\
\hline $\mathrm{N}$ & Cooked root tubers & \multicolumn{2}{|l|}{$\mathrm{R}$} & & 1 & & + & \\
\hline \multicolumn{9}{|c|}{ EUPHORBIACEAE } \\
\hline \multicolumn{5}{|c|}{ Alchornea cordifolia (Schumach. \& Thonn.) Müll.Arg.; Luunze; +; 043214} & 5 & 0.12 & & $M, C$ \\
\hline M & Fever & $\mathrm{L}$ & Crushed leaves & Dermal & 1 & & + & \\
\hline M & Skin disease (furuncle) & $\mathrm{F}$ & Directly consumed & Oral, swallowed in whole & 1 & & + & \\
\hline M & Toothache & L & $\begin{array}{l}\text { Decoction with Psidium guajava and Canarium } \\
\text { schweinfurthii }\end{array}$ & Rinsed in mouth & 3 & & + & \\
\hline \multicolumn{5}{|c|}{ Euphorbia cotinifolia L.; - (*); F11-2000 to F11-2004 } & 1 & 0.02 & & C \\
\hline \multicolumn{2}{|c|}{$\mathrm{O}$} & $P$ & & & 1 & & / & \\
\hline \multicolumn{4}{|c|}{ Euphorbia hirta L.;- (*); 042704} & & 1 & 0.02 & & $M, C$ \\
\hline M & Eye disease & Ms & Latex from sprout & Applied to eye & 1 & & + & \\
\hline \multicolumn{5}{|c|}{ Euphorbia pulcherrima Willd. ex Klotzsch;; 042700} & 3 & 0.07 & & $\mathrm{M}, \mathrm{C}$ \\
\hline M & Childhood disease (blood loss) & & With Maesa sp. & & 1 & & / & \\
\hline M & Inflammation & Ms & Latex from sprout & Dermal & 1 & & / & \\
\hline $\mathrm{O}$ & & $P$ & & & 1 & & / & \\
\hline \multicolumn{5}{|c|}{ Euphorbia thymifolia L.; Mayene mankombo; - (*); 042692} & 2 & 0.05 & & $M, C$ \\
\hline M & Eye disease & Ms & Latex from sprout & Applied to eye & 1 & & - & \\
\hline M & Childhood disease (Diarrhoea) & P & Decoction & $\begin{array}{l}\text { Oral, drunken by mother, cleans } \\
\text { breast milk }\end{array}$ & 1 & & - & \\
\hline \multicolumn{4}{|c|}{ Jatropha curcas L.; Mpuluka; *; F10-1959 } & & 4 & 0.10 & & $M, C$ \\
\hline N & Cooked fruits & $\mathrm{F}$ & Cooked & & 1 & & - & \\
\hline M & Toothache & $\mathrm{L}$ & Decoction & Rinsed in mouth & 3 & & + & \\
\hline \multicolumn{5}{|c|}{ Neoboutonia melleri (Müll.Arg.) Prain; Kiunze, Luunze, +; 043156} & 3 & 0.07 & & C \\
\hline M & Diabetes & $\mathrm{R}$ & Decoction & Oral or enema & 1 & & - & \\
\hline M & Diarrhoea & $\mathrm{R}$ & Decoction & Oral or enema & 1 & & - & \\
\hline M & Abdominal pain & $\mathrm{L}$ & Crushed with salt & Oral & 1 & & o & \\
\hline \multicolumn{5}{|c|}{ Ricinus communis L.; Mamonoa; ;;042668 } & 3 & 0.07 & & $\mathrm{M}, \mathrm{C}$ \\
\hline M & Nausea & $\mathrm{L}$ & Cold water extract of crushed leaves & Oral & 1 & & + & \\
\hline M & Headache & $\mathrm{L}$ & crushed leaves & Applied to head, compress & 3 & & + & \\
\hline
\end{tabular}


Table 1 Overview of the documented useful plant species and their uses sorted by plant families (Continued)

HYPERICACEAE

Harungana madagascariensis Lam. ex Poir.; Ntunu; +; 043193

D Yellow or orange dye

Cold water extract

M Yellow fever

Decoction with e.g. Erythrina abyssinica

M Hepatitis

B Decoction with e.g. Erythrina abyssinica

Psorospermum febrifugum Spach; Nlengula, Kilengula, Pau preto; +;042683

$\begin{array}{llll}\text { M Leprosy } & \text { R } & \text { Crushed root } \\ \text { M } & \text { Fertility } & & \text { Decoction } \\ \text { M } & \text { Skin disease } & \text { B } & \text { Crushed bark } \\ \text { M } & \text { Childhood disease (Baço) } & & \end{array}$

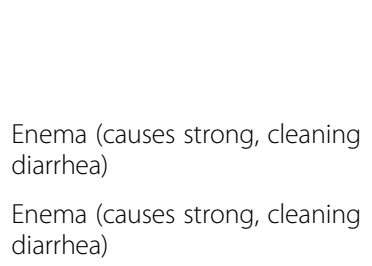

0.12

$M, C$

diarrhea)

Dermal

Oral

Dermal

IRIDACEAE

Eleutherine cf. bulbosa (Mill.) Urb.; - $\left(^{*}\right)$; 043138

O

LAMIACEAE

Alvesia rosmarinifolia Welw.; +; 042670
M Anaemia
L Crushed leaves

Clerodendrum formicarum Gürke; Lomba a mvula (pequena); +; 042662
$M \quad$ Yellow urine
L Infusion
M Childhood disease (Abdominal pain)
L, R Infusion

Hyptis suaveolens (L.) Poit.; Kinsaquati; - (*); 043097
M Fever
L Infusion
indet:; 043148
M Eye disease
Liquid from crushed material

Leonotis sp.; Kakenginzongo; F08-1805

$$
\text { M General L Infusion }
$$

Ocimum gratissimum L.; Mazudizudi, + (as var.); 042719

M Fever, Malaria

M Backache, Rheumatic pain

L $\quad$ With Ageratum conyzoides and D. ambrosioides

Infusion of crushed leaves

With Milletia versicolor, D. ambrosioides and

Persea americana

Dermal

Oral

Oral or enema

Inhalation, steam bath

Applied to eye

Oral

Oral or steam bath

Steam bath 
Table 1 Overview of the documented useful plant species and their uses sorted by plant families (Continued)

\begin{tabular}{|c|c|c|c|c|c|c|c|c|}
\hline M & Diarrhoea & & $\begin{array}{l}\text { With D. ambrosioides, Kafuke (Asteraceae indet.), } \\
\text { M. myristica, X. aethiopica }\end{array}$ & & 1 & & + & \\
\hline M & Respiratory disease & L & Infusion of crushed leaves & Oral or steam bath & 4 & & + & \\
\hline \multicolumn{5}{|c|}{ Vitex madiensis subsp. madiensis; Mafilu, Mfilumfilu; +; 042773} & 10 & 0.24 & & M \\
\hline M & Backache & L & Infusion & Oral & 3 & & - & \\
\hline M & Childhood disease (Gota) & L & $\begin{array}{l}\text { Infusion, sometimes with Gardenia ternifolia } \\
\text { and Smilax anceps }\end{array}$ & & 2 & & o & \\
\hline M & Potency, Strengthening (for women and men) & & Infusion & Oral & 1 & & + & \\
\hline T & & L & & & 1 & & - & \\
\hline $\mathrm{N}$ & Fresh fruits & $\mathrm{F}$ & & & 2 & & + & \\
\hline Fo & & & Pasture for cattle & & 1 & & - & \\
\hline \multicolumn{9}{|c|}{ LEGUMINOSAE } \\
\hline \multicolumn{3}{|c|}{ Albizia sp.; Mulu, Muanse; 043102} & & & 2 & 0.05 & & \\
\hline M & Headache & R & Liquid from crushed root & Applied into nose & 1 & & & \\
\hline M & Diarrhoea & B & Cold water extract from crushed bark & Enema & 1 & & & \\
\hline \multicolumn{5}{|c|}{ Bauhinia variegata $L . ;-(*) ; F 11-2064$} & 1 & 0.02 & & $M, C$ \\
\hline \multicolumn{3}{|l|}{$\mathrm{O}$} & & & 1 & & / & \\
\hline \multicolumn{4}{|c|}{ Cajanus cajan (L.) Millsp.; Wandu; *; 043216} & & 2 & 0.05 & & $M, C$ \\
\hline M & Childhood disease (Gota) & L & Liquid from crushed leaves & Applied into nose and eyes & 1 & & o & \\
\hline N & Cooked fruits & $\mathrm{F}$ & & & 1 & & & \\
\hline \multicolumn{5}{|c|}{ Calopogonium mucunoides Desv.; *; 042672} & 1 & 0.02 & & C \\
\hline Ot & Soil fertility & $P$ & Planted in crop area & & 1 & & / & \\
\hline \multicolumn{5}{|c|}{ Canavalia cf. gladiata (Jacq.) DC.; Nzimamanu; - (*; origin undeterminated); F01-1142 to F01-1144 } & 4 & 0.10 & & $\mathrm{M}, \mathrm{C}$ \\
\hline $\mathrm{R}$ & Protection against mischief & $F, P$ & $\begin{array}{l}\text { (A) Fruit hung around wrist of big children prevents defamation } \\
\text { (B) Plant at the edge of field protects it against influence of bad } \\
\text { neighbours }\end{array}$ & & 3 & & + & \\
\hline M & Childhood disease & $\mathrm{F}$ & Fruit hung around wrist of crying babies & & 1 & & - & \\
\hline \multicolumn{5}{|c|}{ Desmodium velutinum (Willd.) DC; Malamalama; +; 044091, 044092} & 1 & 0.02 & & - \\
\hline M & Headache & $\mathrm{L}$ & Crushed leaves & Dermal, applied to head & 1 & & - & \\
\hline \multicolumn{5}{|c|}{ Eriosema glomeratum (Guill. \& Perr.) Hook.f;; Zila wando; +; 043170} & 1 & 0.02 & & C \\
\hline M & Diarrhoea & L & Directly consumed & Oral & 1 & & o & \\
\hline \multicolumn{5}{|c|}{ Erythrina abyssinica DC.; Mulungulungu, Mungomangoma, Nlungwa kwma; +; 042666, 043211} & 10 & 0.24 & & $M, C$ \\
\hline M & Hepatitis & B & $\begin{array}{l}\text { (A) Infusion (B) decoction with i.a. Harungana madagascariensis } \\
\text { (C) Cold water extract from crushed bark }\end{array}$ & $\begin{array}{l}\text { (A, C) Oral (B) Enema, causes } \\
\text { cleaning diarrhoea }\end{array}$ & 7 & & o & \\
\hline
\end{tabular}


Table 1 Overview of the documented useful plant species and their uses sorted by plant families (Continued)

\begin{tabular}{|c|c|c|c|c|c|c|c|c|}
\hline M & Yellow fever & B & (A) Infusion (B) decoction with i.a. Harungana madagascariensis & $\begin{array}{l}\text { (A) Oral (B) Enema, causes cleaning } \\
\text { diarrhoea }\end{array}$ & 2 & & o & \\
\hline M & Typhoid fever & B & Infusion & Oral & 2 & & - & \\
\hline M & Fertility & B & Decoction with sugar & Oral & 1 & & + & \\
\hline M & Backache & & & & 1 & & o & \\
\hline \multicolumn{3}{|c|}{ indet.; Monguenia; 043096} & & & 3 & 0.07 & & \\
\hline M & Backache & B & Crushed bark & Enema & 1 & & & \\
\hline M & Diarrhoea & R & Cold water extract & Enema & 1 & & & \\
\hline M & Abdominal pain & R & Cold water extract & Enema & 1 & & & \\
\hline \multicolumn{2}{|c|}{ indet.; Musuemba; 043103} & & & & 2 & 0.05 & & \\
\hline $\mathrm{D}$ & Dye & & & & 1 & & & \\
\hline Ot & Fish poison & & & & 1 & & & \\
\hline & t.; Musoshi; 043133 & & & & 1 & 0.02 & & \\
\hline M & Burns & L & Balm from ash mixed with palm oil & Dermal & 1 & & & \\
\hline \multicolumn{2}{|c|}{ Inga sp.; Banana makako, Caseleira; 043101} & & & & 5 & 0.12 & & \\
\hline $\mathrm{N}$ & Fresh fruits & $\mathrm{F}$ & & & 2 & & & \\
\hline $\mathrm{Fu}$ & & W & For charcoal & & 3 & & & \\
\hline \multicolumn{3}{|c|}{ Millettia cf. versicolor Baker; Pau ferro, Mbota, Mbandu; +; 043220} & & & 11 & 0.27 & & $M, C$ \\
\hline $\mathrm{Fi}$ & & $\mathrm{B} / \mathrm{BF}$ & E.g. used to tie off leg & & 1 & & - & \\
\hline M & Measles & B & & Tie around wrist & 2 & & - & \\
\hline M & Backache and rheumatic pain & L & $\begin{array}{l}\text { Decoction with D. ambrosioides, O. gratissimum } \\
\text { and Persea americana }\end{array}$ & Steam bath & 1 & & o & \\
\hline $\mathrm{H}$ & & W & E.g. to produce mortars & & 5 & & + & \\
\hline $\mathrm{Fu}$ & & W & For charcoal production & & 2 & & + & \\
\hline \multicolumn{3}{|c|}{ Senna alata (L.) Roxb.; *; 042691} & & & 3 & 0.07 & & $M, C$ \\
\hline M & Skin disease & L & Crushed leaves & Dermal & 1 & & + & \\
\hline M & Leprosy & L & Balm from roasted leaves and oil & Dermal & 1 & & + & \\
\hline M & Headache & L & Crushed leaves & Compress around head & 1 & & + & \\
\hline \multicolumn{3}{|c|}{ Senna occidentalis (L.) Link; Maniokanioka; *; 044093} & & & 6 & 0.15 & & M \\
\hline M & Abdominal pain & $R, F$ & Cold water extract & $\begin{array}{l}\text { Oral, suitable for persons aged } 6 \text { years } \\
\text { and up }\end{array}$ & 4 & & + & \\
\hline M & Eye disease (e.g. parasites) & L & Liquid from crushed leaves & Applied to eye & 4 & & + & \\
\hline
\end{tabular}


Table 1 Overview of the documented useful plant species and their uses sorted by plant families (Continued)

Tephrosia vogelii Hook.f.; +; 043209

Cold water extractfrom crushed leaves

0.02

Ot Fish poison

LORANTHACEAE

Phragmanthera sp.; Kinama, Nama; F2399; 043159

M Fertility

In combination

0.05

\section{MALVACEAE}

Adansonia digitata L.; Kibaba; +

$\begin{array}{llll}\mathrm{H} & & \text { W } & \\ \mathrm{N} & \text { Fresh/dried fruit } & \text { F } & \text { Fresh or dried pulp or juice } \\ \mathrm{Fu} & \text { Charcoal } & \text { W } & \end{array}$

Cola acuminata (P.Beauv.) Schott \& Endl.; Coleira; +; F06-1664

N Fresh/dried fruit

S Seeds eaten to accompany alcoholic drinks.

To alleviate the bitter taste, fruits are sometimes

put into termite nests prior to consumption.

Gossypium barbadense L.; Algodeiro, Husu; - $\left(^{*}\right)$; 042775
M Abdominal pain
Decoction
M Earache
(A) Boil unripe fruit and apply juice into ear (B)
Rub unripe fruit in hands and stick into ear

Oral

Sida acuta Burm.f.; Lumzumzu; - $\left(^{*}\right)$; 042791
M Hepatitis
M Malaria
M Joint swelling, Build-up of fluid

(A) Liquid from crushed leaves (B) Cold water extract from crushed leaves

L $\quad$ Cold water extract from crushed leaves

Triumfetta cordifolia A.Rich.; Kingongi, Luvunga (PI. Mpunga); + (var. tomentosa); 044030
$\mathrm{Fi}$
M Pregnancy
Decoction

Triumfetta rhomboidea Jacq; Ginsunsu branco; +; 042835

BF

Urena lobata L.; Makolokosso, Gingonge, Ginsunsu; +; 042697

\section{Fertility}

With Kafuke (Asteraceae indet.)

M Childhood disease (general weakening)

Enema

Enema

Enema, to "clean" fetus

(A) Applied to eye (B) enema

Enema

$+$

0.10

$+$

0.02

0.12 
Table 1 Overview of the documented useful plant species and their uses sorted by plant families (Continued)

MELASTOMATACEAE

Tristemma mauritianum J.F. Gmel.; +; 042665

$\mathrm{N} \quad$ Fresh fruit

0.05

MELIACEAE

Azadirachta indica A.Juss.; Neem; - $-^{*}$
M General
Directly consumed or infusion

Oral

0.02

$M, C$

MORACEAE

Artocarpus altilis (Parkinson ex F.A.Zorn) Fosberg; Fruta Pão; -(*); 042674, F19-2542

\begin{tabular}{|c|c|}
\hline N Cooked fruit & $\mathrm{F}$ \\
\hline Fo & $F, L$ \\
\hline
\end{tabular}

MYRTACEAE

Psidium guajava L.; Goiabeira; -(*); 042660

$\begin{array}{llll}\text { N Fresh fruit } & \text { F } & \\ \text { M } & \text { Toothache } & \text { L } & \text { Decoction with A. cordifolia and C. schweinfurthii } \\ \text { M } & \text { Diarrhoea } & L & \text { Directly consumed } \\ \text { M Abdominal pain } & \text { A } & \text { L } & \text { Directly consumed }\end{array}$

OrobanchaceAe
Syzygium guineense (Willd.) DC.; Nkizu; +; 043108
M Diarrhoea
R Cold water extract of crushed leaves, sometimes with Hymenocardia acida
M Fertility
R Cold water extract of crushed leaves

OCHNACEAE

Ochna cf. afzelii subsp. mechowiana (O.Hoffm.) N.Robson; Coxianti; 043153
M Abdominal pain
L $\quad$ With X. aethiopica, M. myristica and Kupidi (Piper sp.)
M Backache
With $X$. aethiopica, M. myristica and Kupidi (Piper sp.)

Sopubia lanata Engl.; Diamba dia kana; + (subsp.); 042703

M Gynaecological disorder

M Measles

Suppository from crushed leaves mixed with e.g. garlic

OXALIDACAEAE

Crushed material

Rectal

Dermal or enema

Oral, also during pregnancy
Oxalis latifolia Kunth; Banana folha; - $\left({ }^{*}\right)$; 044096

M Anaemia

L $\quad$ Directly consumed
Rinse in mouth

Oral

Oral

Oral

Oral

o

$0.02 \quad M, C$

0.07

/

/

0.02

/

PASSIFLORACEAE

Passiflora edulis Sims; Maracujá; * 
Table 1 Overview of the documented useful plant species and their uses sorted by plant families (Continued)

\begin{tabular}{|c|c|c|c|c|c|c|c|c|}
\hline N & Fresh fruit & $\mathrm{F}$ & & & 2 & & + & \\
\hline \multicolumn{9}{|c|}{ PHYLLANTHACEAE } \\
\hline \multicolumn{5}{|c|}{ Bridelia cf. ferruginea Benth.; Nkánkati, Muindu, Windu, Nkalakala; +; 043240} & 11 & 0.27 & & M \\
\hline M & Worms & $B, R$ & Cold water extract from crushed plant material & Enema & 3 & & o & \\
\hline M & Abdominal pain & L & Infusion & Oral & 1 & & + & \\
\hline M & Diarrhoea & $B, R$ & Infusion from crushed plant material & Oral & 2 & & + & \\
\hline M & Wounds & $B, R$ & $\begin{array}{l}\text { (A) Crushed material mixed with small amount } \\
\text { of water; (B) Crude bark }\end{array}$ & Applied to wound & 3 & & o & \\
\hline Ot & Tobacco & $F, L$ & Dry and mix with tobacco & & 2 & & - & \\
\hline $\mathrm{D}$ & Red dye & B & & & 1 & & + & \\
\hline \multicolumn{5}{|c|}{ Hymenocardia acida Tul.; Luvete (PI: Mpete); +; 043186} & 7 & 0.17 & & $M, C$ \\
\hline M & Worms & L & With Bridelia ferruginea & Enema & 1 & & + & \\
\hline M & Diarrhoea & $\mathrm{R}$ & With Syzygium guineense & & 1 & & + & \\
\hline M & Skin disease & B & Balm from powder mixed with oil & & 2 & & + & \\
\hline M & Backache & & & & 1 & & o & \\
\hline M & Abdominal pain & $L, B$ & Directly consumed & Oral or enema & 1 & & + & \\
\hline $\mathrm{Fu}$ & Firewood & W & & & 1 & & + & \\
\hline \multicolumn{5}{|c|}{ Hymenocardia ulmoides Oliv.; Nkalangangula; +; 043194} & 4 & 0.10 & & - \\
\hline M & Abdominal pain & L & Directly consumed (young reddish leaves) & Oral & 2 & & o & \\
\hline M & Diarrhoea & L & Directly consumed (young reddish leaves) & Oral & 1 & & + & \\
\hline M & Nausea & L & Directly consumed (young reddish leaves) & Oral & 1 & & - & \\
\hline M & Hunger & L & Directly consumed (young reddish leaves) & Oral & 1 & & - & \\
\hline $\mathrm{H}$ & & W & & & 1 & & + & \\
\hline \multicolumn{9}{|c|}{ PIPERACEAE } \\
\hline \multicolumn{4}{|c|}{ Piper sp.; +; Kupidi } & & 3 & 0.07 & & \\
\hline M & Worms & S & $\begin{array}{l}\text { Infusion with e.g. X. aethiopica, M. myristica } \\
\text { and Ndungu za nzó (Aframomum sp.) }\end{array}$ & Oral & 1 & & & \\
\hline M & Abdominal pain & L & $\begin{array}{l}\text { With } X \text {. aethiopica, M. myristica and Ochna } \\
\text { cf. afzelii subsp. mechowiana }\end{array}$ & & 1 & & & \\
\hline M & Backache & L & $\begin{array}{l}\text { With } X \text {. aethiopica, M. myristica and Ochna } \\
\text { cf. afzelii subsp. mechowiana. }\end{array}$ & & 1 & & & \\
\hline \multicolumn{9}{|c|}{ POACEAE } \\
\hline \multicolumn{5}{|c|}{ Hyparrhenia sp.; Capim, Maxinde, Musoki; 043992} & 6 & 0.15 & & \\
\hline $\mathrm{H}$ & & L & For roofing, also for walls & & 4 & & & \\
\hline
\end{tabular}


Table 1 Overview of the documented useful plant species and their uses sorted by plant families (Continued)

Fu Firelighter

Fo

Imperata cylindrica (L.) Raeusch.; Kindonga; +; 044098
M Diabetes
M Burns
Rh
Decoction
Balm (with palm oil)
For roofing

Setaria megaphylla (Steud.) T.Durand \& Schinz; Makangaya Madianga; +; 043246

Fo

$\mathrm{L} \quad$ For pigs and rabbits

Primulaceae

Maesa sp.; Nkambakiana; F07-1771
M Childhood disease (blood loss)
With Euphorbia pulcherrima
M Abdominal pain
M Infertility
With Phragmenthera sp.

RUBIACEAE

Gardenia ternifolia subsp. jovis-tonantis(Welw.) Verdc.; Kidia, Kilemba nzau; 043241

\begin{tabular}{|c|c|c|c|}
\hline M & Childhood disease (Gota) & S & $\begin{array}{l}\text { Directly consumed, sometimes with } \\
\text { S. anceps, V. madiensis }\end{array}$ \\
\hline M & Hernia & $\mathrm{R}$ & Cold water extract \\
\hline M & Measles & $F, B, S$ & (A) Decoction, (B) Crushed seeds \\
\hline M & Diabetes & & \\
\hline $\mathrm{R}$ & Lightning arrester & $P$ & $\begin{array}{l}\text { Planted close to house/ branch placed } \\
\text { on roof }\end{array}$ \\
\hline $\mathrm{R}$ & Protection against bad spells & $P$ & Planted in surroundings of house or field \\
\hline R & Nightmares & $\mathrm{F}$ & Placed under pillow \\
\hline \multicolumn{4}{|c|}{ indet.; Ngolanti, Mamunguamungua; 044021} \\
\hline M & Potency & B & Decoction \\
\hline M & Epilepsy & B & Decoction \\
\hline \multicolumn{4}{|c|}{ Iorinda lucida Benth.; Mazige, Nsiki; +; 043215} \\
\hline M & Abdominal pain & $B, L$ & Infusion or directly consumed \\
\hline M & Diarrhoea & & \\
\hline M & Backache & $B, L$ & Crushed material \\
\hline
\end{tabular}

Oral

Dermal

$\begin{array}{rrr}0.12 & & \text { M, C } \\ & + & \\ & \circ & \\ & + & \\ 0.05 & & M \\ & + & \end{array}$

0.07

(A, B) Oral or Enema

Oral, 2 cups daily

Oral, 2 cups daily

Oral (very bitter)

Dermal, applied to back 
Table 1 Overview of the documented useful plant species and their uses sorted by plant families (Continued)

Mussaenda arcuata Poir.; Nsilu-nsilu; +; 042654

M Hepatitis $L \quad$ Crushed leaves

$M \quad$ Skin disease $\quad \mathrm{L} \quad$ Crushed leaves

Sarcocephalus latifolius (Sm.) E.A.Bruce; Kelolo, Kilolwa grande; +; 043154

\begin{tabular}{|c|c|c|c|}
\hline M & Abdominal pain & R & $\begin{array}{l}\text { (A) Cold water extract or decoction (leave in water for } \\
1 \text { h) (B) directly consumed }\end{array}$ \\
\hline M & Worms & $R, B$ & (A) Directly consumed (B) cold water extract (C) decoction \\
\hline M & Potency & $\mathrm{R}$ & (A) Directly consumed (B) Decoction \\
\hline M & Pains & $\mathrm{R}$ & (A) Directly consumed (B) Decoction \\
\hline M & Typhoid fever & R & Decoction \\
\hline M & Birth & $\mathrm{R}$ & Cold water extract \\
\hline M & Respiratory disease & B & Directly consumed \\
\hline $\mathrm{Fu}$ & Firewood & W & \\
\hline $\mathrm{R}$ & Protection & $\mathrm{F}$ & Put on baby \\
\hline $\mathrm{T}$ & & L & \\
\hline
\end{tabular}

RUTACEAE

Zanthoxylum gilletii (De Wild.) P.G.Waterman; Ndansia tenga; +; F15-2398, F15-2396
M Pregnancy
L Cooked, young leaves, strengthing

SMILACACEAE

Smilax anceps Willd.; Gipolo, Mpolo, Mukulu; +; 042702
$M$ Cough
Infusion
M Gota
$L \quad$ With V. madiensis and G. ternifolia
$L, R \quad$ Decoction
M Epilepsy

SOLANACEAE

Datura metel L.; *; 042714
Ot Intoxicant
M Rheumatism
Burn
M Inflammation
With other species
Crushed leaves

Solanum aculeastrum Dunal; Mabumi, Gituno; + (var.); 043132
M Childhood disease (Baço)
F, B Decoction

Solanum americanum Mill.; Lundumbo, Ndumbo; *; 043164
$\mathrm{N}$ Cooked leaves
Cooked and eaten as a vegetable
M Abdominal pain
Cold water extract of crushed leaves

\section{Dermal \\ Dermal}

(A) Oral or enema, (B) Oral

$(A, B)$ Oral (C) Enema

(A) Oral (B) Oral or enema

(A) Oral (B) Oral or enema

Oral

Oral

Oral

Oral, mixed into meals

Oral

0.07
0
0

Bath or enema

$\begin{array}{llll}0.07 & & & M, C \\ & + & \\ & - & \\ & + & \\ & + & \\ 0.05 & & & M, C \\ & - & \\ 0.07 & & & M \\ & - & \\ & - & \\ & 0 & \end{array}$

Inhalation

Dermal

Enema or oral

Oral 
Table 1 Overview of the documented useful plant species and their uses sorted by plant families (Continued)

\begin{tabular}{|c|c|c|c|c|c|c|c|c|}
\hline \multicolumn{4}{|c|}{ Solanum mauritianum Scop.; Daniele; *; 044100} & & \multirow{2}{*}{$\begin{array}{l}1 \\
1\end{array}$} & \multirow[t]{2}{*}{0.02} & & \multirow[t]{2}{*}{ M } \\
\hline M & Cough & $\mathrm{L}$ & Decoction & Steam bath or inhalation & & & - & \\
\hline \multicolumn{5}{|c|}{ Solanum nigrum L.; Gizue, Lundumbo, Windangonge; *; 043139} & 3 & 0.07 & & $\mathrm{M}, \mathrm{C}$ \\
\hline M & for Babys & $\mathrm{L}$ & Sap from crushed leaves & $\begin{array}{l}\text { Few drops daily applied to } \\
\text { umbilical cord }\end{array}$ & 1 & & o & \\
\hline M & Fever & L & Infusion & Oral, suitable for children & 1 & & + & \\
\hline M & Diarrhoea & $\mathrm{R}$ & & & 1 & & + & \\
\hline \multicolumn{9}{|c|}{ URTICACEAE } \\
\hline \multicolumn{4}{|c|}{ Laportea sp.; Kihidi, Hidi; 043157} & & 3 & 0.07 & & \\
\hline M & Cough & $\mathrm{L}$ & Infusion & Oral suitable for children & 2 & & & \\
\hline M & Haemorrhoids & L & Cooked & Oral, eaten with peanuts & 1 & & & \\
\hline \multicolumn{9}{|c|}{ VERBENACEAE } \\
\hline \multicolumn{4}{|c|}{ Lippia multiflora Moldenke; Bulukutu; +; 044101} & & 2 & 0.05 & & $M, C$ \\
\hline T & & L & Infusion & & 2 & & + & \\
\hline \multicolumn{5}{|c|}{ Stachytarpheta cayennensis (Rich.) Vahl; Kalangue; $-\left(^{*}\right) ; 043188$} & 3 & 0.07 & & $\mathrm{M}, \mathrm{C}$ \\
\hline M & Malaria & & Crushed material & Rectal, suppository & 1 & & - & \\
\hline M & Diarrhoea & & Decoction & Oral & 1 & & - & \\
\hline M & Joint swelling, Build-up of fluid & L & Infusion & Oral & 1 & & - & \\
\hline M & Skin disease & $\mathrm{L}$ & Infusion & Oral & 1 & & o & \\
\hline M & Respiratory disease & L & Infusion & Oral & 1 & & - & \\
\hline \multicolumn{9}{|c|}{ VITACEAE } \\
\hline \multicolumn{4}{|c|}{ Cayratia gracilis (Guill. \& Perr.) Suess.; Nlembuzi; +; 043147} & & 1 & 0.02 & & - \\
\hline M & Joint swelling, Build-up of fluid & $\mathrm{L}$ & Crushed leaves & Dermal, applied to swollen joints & 1 & & o & \\
\hline \multicolumn{4}{|c|}{ Cayratia sp.; Hoselia, Uuse; 043166} & & 2 & 0.05 & & \\
\hline M & Anaemia & L & Cooked & Oral & 1 & & & \\
\hline $\mathrm{N}$ & Cooked leaves & L & Cooked and eaten as a vegetable & & 1 & & & \\
\hline \multicolumn{5}{|c|}{ Cissus rubiginosa (Welw. ex Baker) Planch.; Nkokelakai, Mukokelakai; +; 043207} & 4 & 0.10 & & M \\
\hline M & Fever & $\mathrm{L}$ & Crushed leaves added to bathwater & Bath,suitable for children & 1 & & - & \\
\hline M & Childhood disease (Baço) & L & Crushed leaves & Enema & 2 & & - & \\
\hline M & Worms & L & Crushed leaves & Enema & 1 & & - & \\
\hline M & Rheumatism, leg pain & L & Decoction & Enema & 1 & & + & \\
\hline \multicolumn{4}{|c|}{ indet:; Dizo dia lunguenia; 043122} & & 1 & 0.02 & & \\
\hline M & Poisoning & St & - & Used to tie off poisoned limb & 1 & & & \\
\hline
\end{tabular}


Table 1 Overview of the documented useful plant species and their uses sorted by plant families (Continued)

XANTHORRHOEACEAE

Aloe buettneri A.Berger; Badianseka; +; F05-1633
$M \quad$ Skin disease
M Potency
M Fertility
M Worms
M Backache
M Respiratory disease
M Blood-purifying
N Cooked leaves

Sap from cut leaf
Decoction
Decoction
Decoction
Decoction
Directly consumed or decoction
Directly consumed
Eaten with beans as a vegetable

Dermal
Oral or Enema
Enema
Enema

Oral, contains many vitamins

ZINGIBERACEAE

Aframomum alboviolaceum (Ridl.) K.Schum.; Ginguenga da queimada, Masunjá.

Mansassa, Dimbomboa; +; F19-2561, F01-1105
$N \quad$ Fresh fruit
M Yellow fever
M Headache
Rh Crushed rhizome
M Hepatitis
M Respiratory disease
Sap from chewed material
Rh
M Epilepsy
Rh With root of $A$. senegalensis

Enema

Applied to eyes

Aframomum sp.; Ndungu za nzó; F18-2534

$M$ Worms S Infusion with i.e. Kupidi (Piper sp.)
M. myristica and X. aethiopica

M. myristica and X. aethiopica

Zingiber officinale Roscoe; Gengibre, Gibidi; - $\left(^{*}\right)$

\begin{tabular}{lllll} 
& 14 & 0.34 & & $M$ \\
& & & & \\
& 9 & & + & \\
Enema & 1 & & - & \\
Applied to eyes & 1 & & + & \\
& 1 & & - & \\
& 1 & & + & \\
Bath or Enema & 1 & & - \\
& 2 & 0.05 & \\
Oral & 2 & & \\
& 3 & 0.07 & \\
& 2 & & + \\
& 1 & & + \\
& 1 & & 0 \\
\hline
\end{tabular}

M Respiratory disease

$\mathrm{Rh}$

M Fever

Ot

As condiment in palm wine "Maruwu"

Legend: All species identified until family level are listed with scientific and local name and details to representation in Angolan checklist [31]: " + " listed, * listed as naturalised; (*) not listed in checklist, but known to be naturalised in Angola. If herbarium specimens were entered into Herbarium Dresdense, the codes are listed in the table. If it wasn't possible to prepare specimens or if the material was damaged during

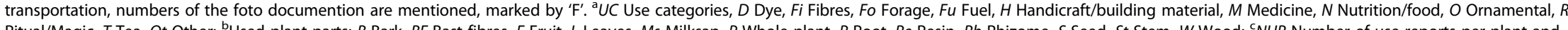
Ritual/Magic, $T$ Tea, Ot Other; ' Used plant parts: $B$ Bark, BF Bast fibres, $F$ Fruit, $L$ Leaves, Ms Milksap, $P$ Whole plant, $R$ Root, Re Resin, Rh Rhizome, $S$ Seed, $S t$ Stem, $W$ Wood; ' $N U R$ Number of use-reports per plant and citations per usage; ${ }^{\mathrm{d} C l}$ Cultural Importance Index; ${ }^{~} L$ Comparision to [44] for medical uses or to [4-6] for other than medical uses: " + "use listed; "o" similar use listed; "-" use not listed; "/" species not listed; f ${ }^{\circ} B$ Result of PubMed-Database-Inquiry in June 2015: (M) medical studies performed, (C) chemical analysis performed 
Table 2 Overview of the ten species with highest number of use-reports (NUR) and cultural importance index (Cl)

\begin{tabular}{llllll}
\hline Species & NUR & UC & SUC & PP & Cl \\
\hline Annona senegalensis & 16 & 3 & 10 & 4 & 0,39 \\
Sarcocephalus latifolius & 16 & 4 & 10 & 5 & 0,39 \\
Aframomum alboviolaceum & 14 & 2 & 6 & 3 & 0,34 \\
Ocimum gratissimum & 11 & 1 & 6 & 1 & 0,27 \\
Millettia cf. versicolor & 11 & 4 & 5 & 4 & 0,27 \\
Bridelia cf. ferruginea & 11 & 3 & 6 & 4 & 0,27 \\
Dysphania ambrosioides & 10 & 1 & 9 & 1 & 0,24 \\
Vitex madiensis subsp. madiensis & 10 & 4 & 6 & 2 & 0,24 \\
Erythrina abyssinica & 10 & 1 & 5 & 1 & 0,24 \\
Gardenia ternifolia subsp. jovis-tonantis & 10 & 2 & 7 & 5 & 0,24
\end{tabular}

Legend: UC Number of use categories, SUC Number of sub-categories, $P P$ different plants parts used

direct consumption of the fresh plant material (50 citations), macerations (27 citations) and other methods, such as burning or drying of the material (4 citations).

Treatments are mostly accomplished through the enteral route by oral intake (139 citations). Rectal drug administration is also common (68 citations), especially by enema. Other methods documented include inhalation (21 citations), ophthalmic drugs (13 citations) and the uptake through oral mucous, e. g. by rinsing (12 citations). Nasal ( 2 citations) and otic (1 citation) treatments were less frequently cited.

As stated before, literature comparison was carried out for all species determined to species level, to emphasize hitherto unknown use-reports. Figure 4 shows the percentage of species whose use for a specific disease pattern was new to the studied literature. The percentage of new species was especially high in categories linked to a specific disease, such as measles, typhoid fever and epilepsy; whereas it was lower for unspecific symptoms i.e. abdominal pain.

\section{Food plants}

During our study, we recorded 53 use-reports for 23 species that are used as food plants. The most commonly cited species are Aframomum alboviolaceum (9 citations), Landolphia owariensis (6 citations), Crassocephalum rubens (5 citations), Pteridium centrali-africanum (4 citations), and Psidium guajava (4 citations).

Fruits are the most frequently used plant parts for nutrition; they are mostly eaten raw (32 citations) or less often cooked or heated in hot water before consumption (6 citations). 13 use-reports mention cooked leaves that are used as a vegetable. Cooked root tubers or raw stems are both documented in only one use-report.

Although the documented species might be found naturalized within the disturbed savannahs of the region, in some cases we also observed cultivation, e.g. for Dacryodes edulis, Canarium schweinfurthii, Cajanus cajan, Artocarpus altilis, Psidium guajava and Passiflora edulis.

Not all plants were incorporated into diets under normal conditions. For example, the taste of Pteridium centrali-africanum fronds was described as rather unpleasant by some of the respondents. For Crassocephalum rubens informants mentioned that they know the leaves are edible and can be used as a vegetable, but do not use them as long as other leafy vegetables such as Manihot esculenta or Amaranthus spp. leaves are available. Similar explanations were documented for the root tubers of Dioscorea dumetorum. Food plants such as these, which are mainly consumed during times of food shortage are often referred to as famine foods [46].

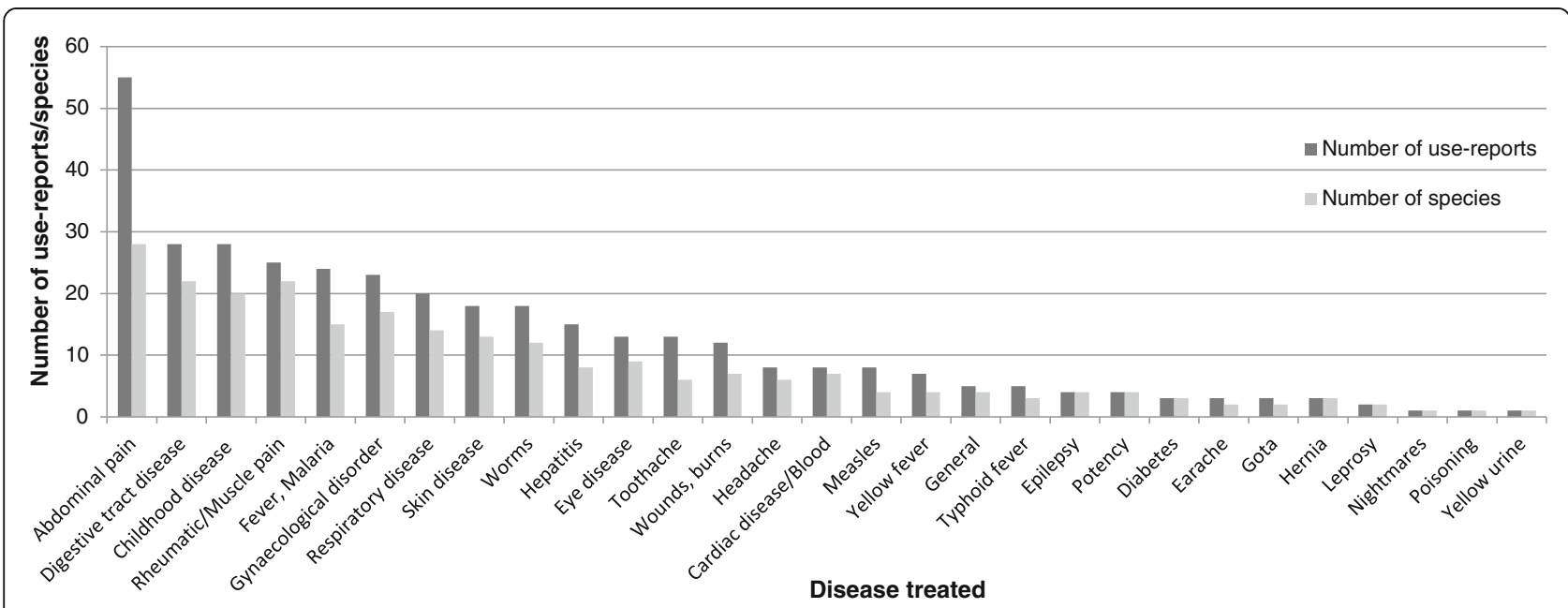

Fig. 3 Analysis of medical use-reports ordered by disease or disease pattern. Number of species and use-reports for different disorders 


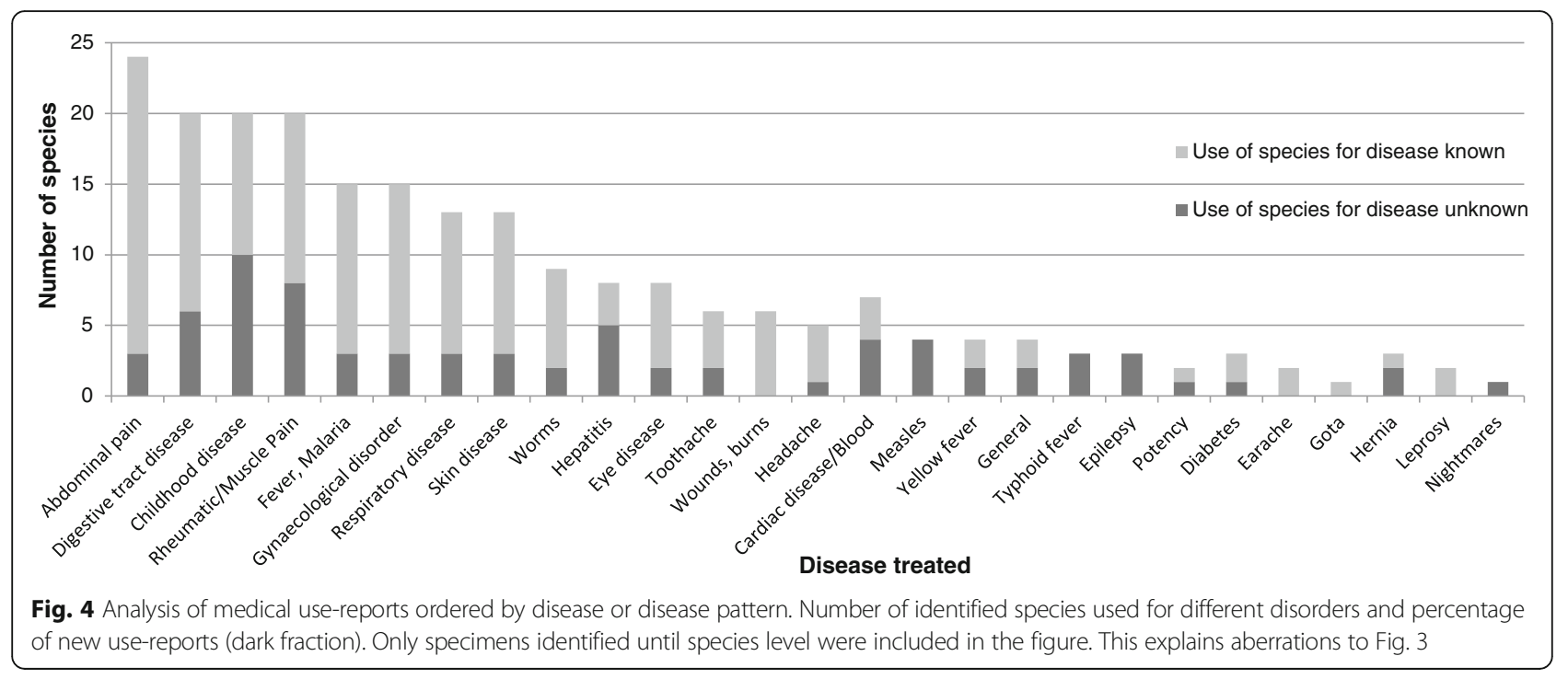

It has already been shown in other studies for subSaharan Africa that some fruits are not harvested in great amounts but rather consumed as a 'snack' while spending time in the field [9, 47]. The fruits of Annona senegalensis, Anisophyllea quangensis, Vitex madiensis subsp. madiensis, Tristemma mauritianum and the stem of Costus afer fit to this description.

In contrast, the fruits of Landolphia owariensis, Canarium schweinfurthii, Dacryodes edulis, Adansonia digitata, Psidium guajava, Passiflora edulis and Aframomum alboviolaceum were sometimes harvested in greater quantities and sold at local markets. Except for Landolphia owariensis, Adansonia digitata and Aframomum alboviolaceum, these plants are also in cultivation, but may have been abandoned or escaped, and are now growing in the surroundings of the villages and fields.

\section{Other uses}

As stated above, other use categories were mentioned less frequently in comparison to medicinal and food plants (in total 55 citations, $17.2 \%$ ). Still, the citations in these categories cover a wide field of different applications, including e. g. fibres and dyes, fuel, building material, ritual and ornamental uses. About $60 \%$ of citations in these use-categories have already been documented in literature about local plant use. Percentage of already known citations is especially high (100 \%) in the categories dyes ( 2 species), fuels (4 species) and handicraft/building material (6 species), which include many woody species. The highest amount of hitherto unknown plant uses was documented in the use-category 'Ornamental', where four of five species haven't been found in the literature used for comparison. Presumably, this is due to the fact that many ethnobotanical studies do not include ornamental uses.

\section{Discussion}

Although savannahs are not the potentially natural vegetation in the study area, they are of high importance to Bakongo people. The 122 documented species are used for diverse applications, ranging from medical treatments and handicrafts to ritual uses. The anthropogenic disturbance of the potential natural vegetation in the area has already been documented in 1970 by Barbosa [19], the incorporation of the plants into livelihood strategies is therefore representing the expectations.

We documented a high amount of use-reports that were new to the literature used for comparison $(34.0 \%$ of citations). Still, the documentation of useful plants in Uíge cannot be considered completed. As time for fieldwork was limited, some plants couldn't be identified until family level and were therefore not included in the documentation. Also, to obtain reliable data, we spatially restricted the study area in order to include a variety of informants with different backgrounds, age and gender from every village. The high percentage of unknown plant uses emphasizes the potential of the study area for further ethnobotanical findings and can be considered as a basis for future research in the region.

\section{Medicinal plants}

The high amount of medical use-reports $(71 \%)$ indicates that plants still play a crucial role in rural health care. This impression is strengthened by the diversity of illnesses treated with plant preparations. As already mentioned before, this is probably due to the difficult access to medical care.

The results regarding preparation and administration of plant remedies are in line with other ethnobotanical works. Decoctions, raw plant parts and infusions as the most 
common preparation methods and oral administration as the most frequent administration route are documented in many studies for sub-Saharan Africa [47-52].

As mentioned before, $34.0 \%$ of all recorded use-reports were not documented in the literature used for comparison; in the use-category of medicinal plants the percentage of previously unknown use-reports is comparable $(33.8 \%)$. To identify promising targets for further biochemical investigation, we additionally conducted a search in the Pubmed-database [53], as documented in Table 1. For the majority of the species (58.4 \%) both medical and phytochemical studies have already been carried out. Further $24.8 \%$ have been analysed either through medical or phytochemical studies. For 17 species $(16.8 \%)$ the inquiry did not reveal further information. As 14 of these species are used in traditional medicine in Northern Angola, they might be promising targets for further studies (Table 3).

Most of the species with high CI-values have already been evaluated in pharmaceutical or medical surveys. Few studies have been conducted for $V$. madiensis subsp. madiensis. G. ternifolia subsp. jovis-tonantis and A. alboviolaceum. Especially G. ternifolia subsp. jovis-tonantis and A. alboviolaceum might be an interesting object of further investigation, as they are used to treat severe illnesses such as measles, diabetes, yellow fever, hepatitis or epilepsy.

\section{Food plants}

The documentation of food plants does mainly meet the expectations. The prevalence of fresh fruits in this usecategory as well as the phenomena of famine foods and snack plants have already been documented in other

Table 3 Species with medical uses not yet tested pharmacologically. Name and overall cultural importance (Cl) of species are given

\begin{tabular}{ll}
\hline Species & $\mathrm{Cl}$ \\
\hline Alvesia rosmarinifolia & 0.02 \\
Anisophyllea cf. quangensis & 0.05 \\
Cayratia gracilis & 0.02 \\
Desmodium velutinum & 0.02 \\
Gymnanthemum cf. glaberrimum & 0,10 \\
Hymenocardia ulmoides & 0.10 \\
Lannea cf. antiscorbutica & 0.10 \\
Oxalis latifolia & 0.02 \\
Pleiotaxis rugosa & 0,07 \\
Pteridium centrali-africanum & 0.12 \\
Smilax anceps & 0.07 \\
Sopubia lanata & 0.07 \\
Triumfetta cordifolia & 0.10 \\
Vernonella cf. subaphylla & 0.12 \\
\hline
\end{tabular}

studies [9, 46]. Our findings support the widelyrepresented concept that food plants collected in the wild are well incorporated into local livelihood strategies in rural, tropical Africa and may contribute to foodsecurity and socio-economic sustainability $[46,54]$.

Most of the plants reported in this use-category have already been documented as food plants. In the literature used for comparison, only Solanum americanum leaves, Tristemma mauritianum fruits, Costus afer stems, Aloe buettneri leaves and Jatropha curcas fruits have not been listed yet. In the cases of Tristemma mauritianum and Solanum americanum the use as a food plant has been documented in other African regions [54-56]. In contrast, Costus afer is well known as a medicinal plant $[44,57]$, but we found no evidence for a use as 'snack food' in the literature. Chemical analysis of the stem shows that there are no toxic effects to be expected and that the plant is a good source of magnesium and potassium [58]. Similarly, Aloe buettneri is a well-known medicinal plant in Africa, whereas documentation for use as a food plant is scarce; only one publication mentions the use of the flowers in soups [59]. Still, it is known that the leaves of other Aloe species may be cooked as a leafy salad, probably after the green leaf tissue has been peeled off to relieve the bitter taste [59]. In contrast, the use of Jatropha curcas fruits as a food is questionable, as they are known to be toxic [60]. There exist publications about non-toxic accessions of the plant, but as they are mainly documented for Mexico [61, 62], it is unlikely that these accessions are grown in the study area. As this use was only mentioned by one informant, we cannot rule out the possibility of inaccurate information. As already stated in the result section, not all citations referred to plants that are regularly used, so we could make no observations about the consumption of Jatropha curcas fruits.

\section{Other uses}

In comparison to medicinal and food plants, other use categories were mentioned less frequently. The small proportion of use-reports for handicraft and building material or fuels might be attributed to the lack of large trees in degraded landscapes. Other uses often seem to be replaced by industrial products, which might lead to a loss of traditional knowledge. As could be observed in the surroundings of Uíge city, traditional thatching materials are often replaced by corrugated sheet metal. Similarly, the knowledge about natural dyes was still present, although informants stated that they hadn't used them by themselves.

\section{Impact of disturbance and implications for conservation}

The species of highest cultural importance, as listed in Table 2, include no species of special concern. All plants listed are readily available in the region: $A$. senegalensis, 
S. latifolius, A. alboviolaceum, B. ferruginea, V. madiensis subsp. madiensis, E. abyssinica and G. ternifolia subsp. jovis-tonantis belong to the typical vegetation of disturbed savannahs, while O. gratissimum, $M$. versicolor and $D$. ambrosioides are rather found in the transition zones to village outskirts.

It is conspicuous that seven of the ten highest ranked plants are woody species. These values might be attributed to the fact that trees and shrubs offer a greater variety of useable parts than herbaceous plants, e. g. wood and bark $[41,63,64]$ and that woody plants are available throughout the year $[9,65]$. In the context of disturbance vegetation, other reasons might be the greater visibility of trees and shrubs in grasslands as well a better resistance of mature trees to fire in comparison to many herbaceous plants $[9,13]$.

Nevertheless, the amount of annual and perennial herbaceous plants is still high (49.6\%). This number might be surprising, considering that the Hyparrhenium species, which dominate the savannahs of the region, form compact eyries and reach heights up to $2.5 \mathrm{~m}$ and therefore inhibit the growth of small herbaceous plants. From that point of view, anthropological disturbance actually might contribute to a higher biodiversity in anthropogenic savannahs. Grazing, agriculture and small savannah fires can create a mosaic of small-scale habitats with different light conditions and therefore permits the growth of herbaceous, short-lived species and pyrophytic plants [66, 67]. It has been shown that some traditional fire management practices are specifically aimed at the creation of such small-scale habitats (patch-mosaic burning) [68].

Although no such traditional fire management systems are known for the north of Angola, we observed that some of the used species, e. g. Helichrysum mechowianum, Pleiotaxis rugosa, Baccharoides guineensis or Smilax anceps, often grew in recently burnt areas, where light exposure was high. Likewise, the young fronds of Pteridium centrali-africanum were regularly found in recently burnt areas, where proliferation is promoted. Similarly, fruits of Aframomum alboviolaceum were often collected in recently burnt savannahs. This might be related to the fact that a thick grass cover does not only inhibit growth of small herbaceous plants, but also impedes access for plant collection. Two informants explained during semistructured interviews that sometimes grassland is specifically burnt to facilitate access to Aframomum alboviolaceum fruits, which may be sold at local markets. Therefore, even if the above-mentioned species are not critically endangered, their collection may exert high pressure on the ecosystem, especially when large areas are exposed or fires are repeated within short time periods [69]. Besides, for some woody species, e. g. E. abyssinica, B. ferruginea and $S$. latifolius, fire resistance was observed to be severely affected by intensive collection of roots and bark.
Loss of bark may lead to higher vulnerability towards fungal infection and pests, and decrease fire and drought resistance and by that means lead to further reduction of woody species in the area [70, 71].

As can be noticed by those examples, the analysis of plant use and cultural importance of plants may help to understand land use and fire management practices and to develop conservation strategies to ensure the access to useful plants for future generations. However, a complete analysis of that topic must take many other aspects into account, e.g. economic, ritual or agricultural issues.

\section{Conclusion}

Despite the comparably low biodiversity, plants from degraded savannah vegetation in Uíge, Angola, are incorporated in manifold livelihood strategies of the local population. Savannahs are sources of food and medicine; they provide plants for handicraft, forage and fuel as well as ritual and ornamental plants. While some uses, e. g. dyes, fibres and building material often seem to be replaced by industrial products, we recorded a high amount of medicinal plants $(70 \%)$. As access to health care especially in rural areas is difficult, plants still play a vital role in this field.

As a result of the diverse incorporation of plants in livelihood strategies the north of Angola turns into a promising region for further ethnobotanical research. This is underlined by the high amount (34.0\%) of usereports that have not been documented in the literature used for comparison.

Especially in the field of medicinal plants, further investigation is necessary to evaluate the pharmacological potential of the plants and to improve self-medication practises in the future.

Our study highlights 14 species which are especially interesting for pharmacological analysis. It is essential to communicate the results of ethnobotanical and pharmacological research to the rural population. In the framework of the project, the establishment of a botanical garden based on ethnobotanical criteria is envisaged. The presented study encourages those efforts, as it both points out the richness of ethnobotanical knowledge in the region and the importance of plant remedies in rural health care.

The species of highest cultural importance do not include plants of special concern. Still, collection of plants can exert high pressure on the ecosystem, as sometimes fires might be set to improve accessibility of savannahs. In order to conserve the savannahs as a source of useful plants, further studies should take a closer look at plant collection and traditional fire management systems.

\section{Acknowledgements}

The authors would like to thank Dr. Barbara Ditsch, Gerard van der Weerden and Paul Latham for assistance in the identification of selected herbarium specimens. We express our profound gratitude to all local communities involved in this study for sharing their knowledge and experiences. 


\section{Funding}

The fieldwork in Angola was supported by a travel fund from the German Academic Exchange Service (DAAD). These published results were obtained in collaboration with the Instituto Nacional da Biodiversidade e Áreas de Conservação (INBAC) of the Ministério do Ambiente da República de Angola.

\section{Availability of data and materials}

All data are available from the corresponding author. All voucher specimens are deposited in the Herbarium Dresdense (DD) of the Institute of Botany, Technische Universität Dresden, Germany. As soon as suitable conditions are established, parts of the collection will be deposited at Universidade Kimpa Vita, Uíge, Angola.

\section{Authors' contributions}

AG carried out field work, analysed the collected data and drafted the manuscript. ABTN and MF participated in field work and established contact to local people. $T L$ and $C N$ participated in the design of the study and helped to draft the manuscript, TL also supported data analysis. All authors read and approved the final manuscript.

\section{Competing interests}

The authors declare that they have no competing interests.

\section{Consent for publication}

All authors have approved the manuscript and agree with its submission to Journal of Ethnobiology and Ethnomedicine.

\section{Ethics approval and consent to participate}

Not applicable.

\section{Author details}

${ }^{1}$ Institut für Botanik, Technische Universität Dresden, D-01062 Dresden, Germany. ${ }^{2}$ Universidade Kimpa Vita, Province of Uíge, Rua Henrique Freitas No. 1, Bairro Popular, Uíge, Angola.

\section{Received: 8 April 2016 Accepted: 13 September 2016}

\section{Published online: 20 September 2016}

\section{References}

1. Kuedikuenda S, Xavier M. Framework report on Angola's Biodiversity. Republic of Angola: Ministry of Environment; 2009. https://69.90.183.227/ doc/world/ao/ao-nr-04-en.pdf. Accessed 17 Dec 2015.

2. CIA. The World Factbook. https://www.cia.gov/library/publications/theworld-factbook/geos/ao.html. Accessed 30 Jun 2015.

3. Leyens T, Lobin W. Manual de plantas úteis de Angola. Bischöfliches Hilfswerk Misereor e.V., editor. Aachen: MVG-Medienproduktion und Vertriebsges; 2009.

4. Costa EMEF da, Pedro M. Plantas medicinais de Angola. Luanda: Universidade Agostinho Neto, Centro de Botânica; 2013.

5. Latham P, Konda ku Mbuta A. Useful Plants of Bas-Congo Province, Democratic Republic of Congo. 2014

6. Latham P, Konda ku Mbuta A. Some honeybee plants of Bas-Congo Province, Democratic Republic of Congo. 2. 2011.

7. Kembelo K. Quelques plantes medecinales du Bas-Congo et leurs usage. 2003. http://www.ethnopharmacologia.org/prelude/pdf/bibio-hk-61kibungu.pdf. Accessed 06 Mar 2016

8. Makumbelo E, Lukoki L, Paulus J, Luyindula N. Stratégie de valorisation des espèces ressources des produits non ligneux de la savane des environs de Kinshasa: II. Enquête ethnobotanique (aspects médicinaux). Tropicultura. 2008;26:129-34.

9. Urso V, Signorini MA, Tonini M, Bruschi P. Wild medicinal and food plants used by communities living in Mopane woodlands of southern Angola: Results of an ethnobotanical field investigation. J Ethnopharmacol. 2016;177:126-39.

10. Lautenschläger T, Neinhuis C, editors. Riquezas naturais de Uíge - Uma breve introdução sobre o estado atual, a ameaça e a preservação da biodiversidade. Dresden: Technische Uni Dresden; 2014.

11. Posey DA. Indigenous management of tropical forest ecosystems: the case of the Kayapó indians of the Brazilian Amazon. Agrofor Syst. 1985:3:139-58.

12. Towns AM, Ruysschaert $S$, van Vliet $E$, van Andel T. Evidence in support of the role of disturbance vegetation for women's health and childcare in Western Africa. J Ethnobiol Ethnomedicine. 2014;10:42.
13. Phillips O, Gentry AH. The useful plants of Tambopata, Peru: II. Additional hypotheses testing in quantitative ethnobotany. Econ Bot. 1993;47:33-43.

14. Stepp JR. The role of weeds as sources of pharmaceuticals. J Ethnopharmacol. 2004;92:163-6.

15. Stepp JR, Moerman DE. The importance of weeds in ethnopharmacology. J Ethnopharmacol. 2001;75:19-23.

16. Briggs DJ, Smithson P. Fundamentals of Physical Geography. New Jersey: Rowman \& Littlefield; 1986.

17. Peel MC, Finlayson BL, McMahon TA. Updated world map of the KöppenGeiger climate classification. Hydrol Earth Syst Sci. 2007;11:1633-44.

18. Marquardsen H, editor. Angola. 2nd ed. Berlin: Reimer; 1928.

19. Barbosa LAG. Carta fitogeográfica de Angola. Luanda: Instituto de Investigação Científica de Angola; 2009.

20. Kuder M, editor. Angola / Naturraum, Wirtschaft, Bevölkerung, Kultur, Zeitgeschichte und Entwicklungsperspektiven. München: Weltforum-Verl; 1994.

21. Censo 2014. http://censo.ine.gov.ao/xportal/xmain?xpid=censo2014\&xpgid= provincias\&provincias-generic-detail_qry=BOUI=10327871\&actualmenu= 10327871\&actualmenu=10496934. Accessed 26 Nov 2014

22. B Barroso Quiala M. Longoka Kikongo. 1st ed. Luanda: Mayamba Editora; 2013.

23. Kassie GT, Erenstein O, Mwangi W, LaRovere R, Stetimela P, Langyintuo A. Characterization of Maize Production in Southern Africa: Synthesis of CIMMYT/DTMA Household Level Farming System Surveys in Angola, Malawi, Mozambique, Zambia and Zimbabwe. Mexico, D.F.: CIMMYT; 2012. http://dtma.cimmyt.org/index.php/publications/doc view/168characterization-of-maize-production-in-southern-africa-synthesis-of-cimmytdtma-household-level-farming-system-surveys-in-angola-malawimozambique-zambia-and-zimbabwe. Accessed 10 Feb 2015.

24. FAO. Food and Agriculture Organization: Angola Country Programming Framework 2013-2017. 2012. ftp://ftp.fao.org/TC/CPF/Countries/Angola/ CPFAngola.pdf. Accessed 27 Dec 2014.

25. Queza AJ. Sistema de saúde em Angola: Uma proposta à luz da reforma do serviço nacional de saúde em Portugal. Mestrado Integrado em Medicina, Universidade do Porto; 2009 http://repositorio-aberto.up.pt/bitstream/10216/50407/ 2/Sistema\%20Nacional\%20de\%20Sade\%20Angolano\%20e\%20Contributos\%20\%20 Luz\%20da\%20Reforma\%20do\%20SNS\%20Portugus.pdf?. Accessed 30 March 2016.

26. UN Inter-agency Group for Child Mortality Estimation, editor. Levels \& trends in Child Mortality. 2015. http://childmortality.org/files_v20/ download/IGME\%20report\%202015\%20child\%20mortality\%20final.pdf. Accessed 28 Feb 2016.

27. Ministerio da Saúde de Angola. Plano Nacional de Desenvolvimento Sanitário 2012-2025. 2014. http://www.minsa.gov.ao/NerPublicacao.aspx?id=1266. Accessed 17 Dec 2014

28. Exell AW, Mendoca FA. Conspectus florae angolensis. Lisboa: Ministerio do Ultramar, Junta de Investigacoes colonais; 1954.

29. Exell AW, Mendoca FA. Conspectus florae angolensis. Wittnich Carisso L, editor. Lisboa: Ministerio do Ultramar, Junta de Investigacoes colonais; 1937.

30. Exell AW, Mendoca FA. Conspectus florae angolensis. Lisboa: Ministerio do Ultramar, Junta de Investigacoes colonais; 1962.

31. Figueiredo E, Smith G. Plantas de Angola. Pretoria: South African National Biodiversity Institute (SANBI); 2008.

32. Hutchinson J, Dalziel JM. Flora of West Tropical Africa: The British West African territories, Liberia, the French and Portuguese territories South of latitude $18^{\circ} \mathrm{N}$. to Lake Chad, and Fernando Po. 2nd ed. London: Crown agents for Oversea Governments and Administrations; 1954.

33. Hutchinson J, Dalziel JM. Flora of West Tropical Africa: All territories in West Africa South of latitude $18^{\circ} \mathrm{N}$. and to the West of Lake Chad, and Fernando Po. 2nd ed. London: Crown agents for Oversea Governments and Administrations; 1972

34. Hutchinson J, Dalziel JM. Flora of West Tropical Africa: The British West African territories, Liberia, the French and Portuguese territories South of latitude $18^{\circ} \mathrm{N}$. to Lake Chad, and Fernando Po. 2nd ed. London: Crown agents for Oversea Governments and Administrations; 1958.

35. Hutchinson J, Dalziel JM. Flora of West Tropical Africa: All territories in West Africa South of latitude $18^{\circ} \mathrm{N}$. and to the West of Lake Chad, and Fernando Po. 2nd ed. London: Crown agents for Oversea Governments and Administrations; 1963.

36. Hutchinson J, Dalziel JM. Flora of West Tropical Africa: All territories in West Africa South of latitude $18^{\circ} \mathrm{N}$. and to the West of Lake Chad, and Fernando Po. 2nd ed. London: Crown agents for Oversea Governments and Administrations; 1968 
37. Akoègninou A, Burg WJ van der, Maesen LJG van der. Flore analytique du Bénin. Backhuys; 2006

38. Royal Botanic Gardens Kew. Flora Zambesica. 2007. http://apps.kew.org/efloras. Accessed 30 Dec 2015.

39. Royal Botanic Gardens Kew. Kew Herbarium Catalogue. 2014. http://apps. kew.org/herbcat. Accessed 30 Dec 2015.

40. Instituto de Investigação Científica Tropical Portugal. Herbário LISC. 2007. http://maerua.iict.pt/colecoes/herb_simplesearch.php. Accessed 14 Jul 2015.

41. Tardío J, Pardo-de-Santayana M. Cultural importance indices: a comparative analysis based on the useful wild plants of Southern Cantabria (Northern Spain)1. Econ Bot. 2008;62:24-39.

42. Kufer J, Heinrich M, Förther H, Pöll E. Historical and modern medicinal plant uses - the example of the Ch'orti' Maya and Ladinos in Eastern Guatemala. J Pharm Pharmacol. 2005;57:1127-52.

43. Medeiros MFT, da Silva PS, de Albuquerque UP. Quantification in ethnobotanical research : an overview of indices used from 1995 to 2009. Sitientibus Sér Ciênc Biol. 2011:11:211-30.

44. Neuwinger HD. African traditional medicine / a dictionary of plant use and applications. Stuttgart: Medpharm; 2000.

45. Latham P. Scamperdale - Fotostream on flickr. https://www.flickr.com/ photos/36517976@N06/sets/. Accessed 23 Dec 2014.

46. Muller J, Almedom AM. What is "Famine Food"? distinguishing between traditional vegetables and special foods for times of hunger/scarcity (Boumba, Niger). Hum Ecol. 2008;36:599-607.

47. Maroyi A. The gathering and consumption of wild edible plants in Nhema Communal Area, Midlands Province, Zimbabwe. Ecol Food Nutr. 2011;50:506-25.

48. Gail H, Tarryn B, Oluwaseyi A, Denver D, Oluchi M, Charlotte VK, et al. An ethnobotanical survey of medicinal plants used by traditional health practitioners to manage HIV and its related opportunistic infections in Mpoza, Eastern Cape Province, South Africa. J Ethnopharmacol. 2015;171:109-15.

49. Bajin Ba Ndob I, Mengome LE, Bourobou Bourobou H-P, Lossangoye Banfora Y, Bivigou F. Ethnobotanical survey of medicinal plants used as anthelmintic remedies in Gabon. J Ethnopharmacol. 2016;191:360-71.

50. Omwenga EO, Hensel A, Shitandi A, Goycoolea FM. Ethnobotanical survey of traditionally used medicinal plants for infections of skin, gastrointestinal tract, urinary tract and the oral cavity in Borabu sub-county, Nyamira county, Kenya. J Ethnopharmacol. 2015;176:508-14.

51. Ngezahayo J, Havyarimana F, Hari L, Stévigny C, Duez P. Medicinal plants used by Burundian traditional healers for the treatment of microbial diseases. J Ethnopharmacol. 2015;173:338-51.

52. Kankara SS, Ibrahim MH, Mustafa M, Go R. Ethnobotanical survey of medicinal plants used for traditional maternal healthcare in Katsina state, Nigeria. South Afr J Bot. 2015;97:165-75.

53. NCBI. PubMed. http://www.ncbi.n/m.nih.gov/pubmed Accessed 20 Jun 2015.

54. Musinguzi E, Kikafunda JK, Kiremire BT. Utilization of indigenous food plants in Uganda: A case study of south-western Uganda. Afr J Food Agric Nutr Dev. 2006;6:2

55. Oladele Ol. Contribution of indigenous vegetables and fruits to poverty alleviation in Oyo State, Nigeria. J Hum Ecol. 2011;34:1-6.

56. Dansi A, Adjatin A, Adoukonou-Sagbadja H, Faladé V, Yedomonhan H, Odou $D$, et al. Traditional leafy vegetables and their use in the Benin Republic. Genet Resour Crop Evol. 2008:55:1239-56.

57. Chhabra SC, Mahunnah RLA, Mshiu EN. Plants used in traditional medicine in Eastern Tanzania. VI. Angiosperms (Sapotaceae to Zingiberaceae). J Ethnopharmacol. 1993:39(2):83-103.

58. Akpabio UD, Udo UE, Akpakpan AE. Evaluation of phytochemical, proximate and mineral element composition of stem of Costus afer (Bush cane). Asian J Plant Sci Res. 2012;2:607-12.

59. Grace OM, Simmonds MSJ, Smith GF, Wyk AE van. Documented utility and biocultural value of Aloe L. (Asphodelaceae): a review. Econ Bot. 2009;63:167-78.

60. Abdu-Aguye I, Sannusi A, Alafiya-Tayo RA, Bhusnurmath SR. Acute toxicity studies with Jatropha curcas L. Hum Toxicol. 1986;5:269-74.

61. Valdes-Rodriguez OA, Sanchez-Sanchez O, Perez-Vazquez A, Caplan J. The Mexican non-toxic Jatropha curcas L., Food Resource or Biofuel? Ethnobot. Res Appl. 2013;11:001-7.

62. Makkar HPS, Aderibigbe AO, Becker K. Comparative evaluation of non-toxic and toxic varieties of Jatropha curcas for chemical composition, digestibility, protein degradability and toxic factors. Food Chem. 1998;62:207-15.

63. Moerman DE. The medicinal Flora of native North America: an analysis. J Ethnopharmacol. 1991;31:1-42.
64. dos Santos LL, Ramos MA, da Silva SI, de Sales MF, de Albuquerque UP. Caatinga ethnobotany: anthropogenic landscape modification and useful species in Brazil's Semi-Arid Northeast. Econ Bot. 2009;63:363.

65. de Albuquerque UP. Re-examining hypotheses concerning the use and knowledge of medicinal plants: a study in the Caatinga vegetation of NE Brazil. J Ethnobiol Ethnomedicine. 2006;2:30.

66. Fuhlendorf SD, Engle DM. Application of the fire-grazing interaction to restore a shifting mosaic on tallgrass prairie: Shifting mosaic on tallgrass prairie. J Appl Ecol. 2004;41:604-14.

67. Laris P, Wardell DA. Good, bad or "necessary evil"? Reinterpreting the colonial burning experiments in the savanna landscapes of West Africa. Geogr J. 2006;172:271-90.

68. Walters GM. The Land Chief's embers: ethnobotany of Batéké fire regimes, savanna vegetation and resource use in Gabon. London: University College London; 2010.

69. Hauser S, Norgrove L. Slash-and-Burn Agriculture, Effects of. In: Levin SA, editor. Encycl. Biodivers. 2nd ed. Waltham: Academic; 2001. p. 551-62.

70. Stewart K. Effects of bark harvest and other human activity on populations of the African cherry (Prunus africana) on Mount Oku, Cameroon. For Ecol Manag. 2009;258:1121-8.

71. Delvaux C, Sinsin B, Darchambeau F, Van Damme P. Recovery from bark harvesting of 12 medicinal tree species in Benin, West Africa. J Appl Ecol. 2009;46:703-12.

\section{Submit your next manuscript to BioMed Central and we will help you at every step:}

- We accept pre-submission inquiries

- Our selector tool helps you to find the most relevant journal

- We provide round the clock customer support

- Convenient online submission

- Thorough peer review

- Inclusion in PubMed and all major indexing services

- Maximum visibility for your research

Submit your manuscript at www.biomedcentral.com/submit

) BioMed Central 